\title{
Asymptotic Behaviour of Nonlinear Systems
}

\author{
Hartmut Logemann and Eugene P. Ryan
}

1. INTRODUCTION. Qualitative results on the long-term behaviour of dynamical processes are of great importance in the applications of differential equations, dynamical systems, and control theory to science and engineering. Although Lyapunov's famous memoire on the stability of motion (published in 1892 in Russian) was translated into French in 1907 and reprinted in the U.S.A. in 1949 [23], ${ }^{1}$ it was only at the end of the 1950s that scientists in the West began to appreciate, use, and develop further Lyapunov's seminal contributions to stability theory. This contrasted with the pre-eminence Lyapunov's direct method had achieved in the Soviet Union as a major mathematical tool in the context of linear and nonlinear stability problems (see [15]). Today, Lyapunov's direct method is a standard ingredient of the syllabuses of university courses on differential equations, dynamical systems, and control theory taught in mathematics, engineering, and science departments worldwide. With Lyapunov's direct method as exemplar, this paper attempts to provide a self-contained, elementary, and unified approach to the analysis of certain aspects of the asymptotic behaviour of solutions of ordinary differential equations and differential inclusions. As a starting point, we make the simple observation, due to Barbălat [3], that if a function $y:[0, \infty) \rightarrow \mathbb{R}$ is uniformly continuous and integrable, then $y(t)$ necessarily approaches 0 as $t \rightarrow \infty$ (see section 3 for a proof). This result, usually referred to as Barbălat's lemma, was derived in [3] as a tool for the analysis of the asymptotic behaviour of a class of systems of nonlinear secondorder equations with forcing. In the context of an autonomous ordinary differential equation $\dot{x}=f(x)$ with locally Lipschitz $f: \mathbb{R}^{N} \rightarrow \mathbb{R}^{N}$, Barbălat's lemma leads in an entirely elementary manner to the invariance principle of LaSalle, a generalization of Lyapunov's theorem on asymptotic stability. The following sketches this elementary argument. Let $V: \mathbb{R}^{N} \rightarrow \mathbb{R}$ be continuously differentiable and assume that $V_{f}(\xi):=\langle\nabla V(\xi), f(\xi)\rangle \leq 0$ for all $\xi$ in $\mathbb{R}^{N}$. Moreover, assume that $x$ is a bounded solution of the differential equation. Then $V \circ x$ is bounded with derivative $(d / d t) V(x(t))=\left(V_{f} \circ x\right)(t) \leq 0$ for all $t \geq 0$. Therefore, $(V \circ x)(t)$ converges to a finite limit as $t \rightarrow \infty$, from which the integrability of $V_{f} \circ x$ follows. Since $x$ is bounded, the derivative $\dot{x}=f(x)$ is also bounded, and thus $x$ is uniformly continuous. Therefore, we may conclude that the integrable function $V_{f} \circ x$ is uniformly continuous. By Barbălat's lemma, $V_{f}(x(t)) \rightarrow 0$ as $t \rightarrow \infty$, implying that $x(t)$ approaches the zero level set $V_{f}^{-1}(0)$ of $V_{f}$ as $t \rightarrow \infty$. Consequently, the $\omega$-limit set $\Omega(x)$ must be contained in $V_{f}^{-1}(0)$. Combining this with the fact that $\Omega(x)$ is invariant with respect to the flow generated by the differential equation, we see that $x(t)$ approaches the largest invariant subset contained in $V_{f}^{-1}(0)$ : this is LaSalle's invariance principle, which (together with its variants and generalizations) is ubiquitous in the stability theory of differential equations (including control theory) and dynamical systems (see, for example, [1], [13], [16], [18], [19], [20], [28], [31], [32]). The importance of the invariance principle stems from the fact that the conditions on $V$ are less restrictive than those imposed in the classical result of Lyapunov on asymptotic stability

\footnotetext{
${ }^{1}$ It was eventually translated into English by Fuller in 1992 [24], a hundred years after the publication of the original.
} 
(which can be considered as a special case of the invariance principle): in particular, the invariance principle does not require $V_{f}$ to be strictly negative; see section 3 for more details. We mention that the "standard" proof of LaSalle's invariance principle, widespread in the literature (see, for example, [1], [13], [18], [19], [20], [28], [32]), is somewhat different insofar as Barbălat's lemma is not usually invoked. Instead, the convergence of $V(x(t))$ as $t \rightarrow \infty$ is used to conclude that $V$ is constant on $\Omega(x)$, which in turn implies (via a straightforward argument based on the invariance of $\Omega(x)$ ) that $V_{f}(\xi)=0$ for all $\xi$ in $\Omega(x)$. In this paper, we show that suitable generalizations of the first argument (involving Barbălat's lemma) lead to diverse results on asymptotic dynamic behaviour in the more general setting of nonautonomous ordinary differential equations and (autonomous) differential inclusions. Our goal is first to develop a compendium of results pertaining to asymptotic behaviour of functions and constituting generalizations of Barbălat's lemma. We achieve this by elementary arguments based on concepts of meagreness and weak meagreness of functions, which, in conjunction with uniform continuity on particular subsets of $[0, \infty)$, capture certain asymptotic properties of functions $t \mapsto y(t)$ as $t \rightarrow \infty$. This compendium then forms the basis for a unified approach to various results (including generalizations of LaSalle's invariance principle) on asymptotic behaviour of solutions of (nonautonomous) ordinary differential equations and (autonomous) differential inclusions. The paper has a tutorial flavour and, for purposes of illustration, we have included detailed descriptions of three examples.

2. PRELIMINARIES. In order to render the paper essentially self-contained, we first assemble some familiar facts, notation, and terminology. Throughout, $\mathbb{N}$ denotes the set of positive integers, $\mathbb{R}_{+}:=[0, \infty)$, and $\mu$ denotes Lebesgue measure on $\mathbb{R}_{+}$. The Euclidean inner product and induced norm on $\mathbb{R}^{N}$ are denoted by $\langle\cdot, \cdot\rangle$ and $\|\cdot\|$, respectively. Let $x: \mathbb{R}_{+} \rightarrow \mathbb{R}^{N}$ be a Lebesgue measurable function: if $0<p<\infty$ and the function $t \mapsto\|x(t)\|^{p}$ is Lebesgue integrable (respectively, locally Lebesgue integrable, that is, Lebesgue integrable over each compact subset of $\left.\mathbb{R}_{+}\right)$, then we write $x \in L^{p}$ (respectively, $x \in L_{\text {loc }}^{p}$ ); if the function $t \mapsto\|x(t)\|$ is essentially bounded (respectively, locally essentially bounded), then we write $x \in L^{\infty}$ (respectively, $x \in L_{\mathrm{loc}}^{\infty}$ ).

Let $A$ be a nonempty subset of $\mathbb{R}^{N}$, and let $h: A \rightarrow \mathbb{R}^{P}$. For a subset $U$ of $\mathbb{R}^{P}$, $h^{-1}(U)$ denotes the preimage of $U$ under $h$, that is, $h^{-1}(U):=\{\xi \in A: h(\xi) \in U\}$; for notational simplicity, if $u$ belongs to $\mathbb{R}^{P}$, then we write $h^{-1}(u)$ in place of the more cumbersome $h^{-1}(\{u\})$. We recall that $h$ is continuous at a point $\xi_{0}$ of $A$ if, for every $\varepsilon>0$, there exists $\delta>0$ such that $\left\|h\left(\xi_{0}\right)-h(\xi)\right\| \leq \varepsilon$ for all $\xi$ in $A$ with $\left\|\xi_{0}-\xi\right\| \leq \delta$. If $h$ is continuous at $\xi$ for all $\xi$ in a subset $B$ of $A$, then $h$ is said to be continuous on $B$; if $B=A$, then we simply say that $h$ is continuous. The function $h$ is uniformly continuous on a subset $B$ of $A$ if, for every $\varepsilon>0$, there exists $\delta>0$ such that $\left\|h\left(\xi_{1}\right)-h\left(\xi_{2}\right)\right\| \leq \varepsilon$ for all points $\xi_{1}$ and $\xi_{2}$ of $B$ with $\left\|\xi_{1}-\xi_{2}\right\| \leq \delta$; if $B=A$, then we say that $h$ is uniformly continuous. It is convenient to adopt the convention that $h$ is uniformly continuous on the empty set $\emptyset$. If $h$ is scalar-valued (that is, $P=1$ ), then $h$ is lower semicontinuous if $\liminf _{\xi^{\prime} \rightarrow \xi} h\left(\xi^{\prime}\right) \geq h(\xi)$ for all $\xi$ in $A$, while $h$ is upper semicontinuous if $-h$ is lower semicontinuous.

The Euclidean distance function for a nonempty subset $A$ of $\mathbb{R}^{N}$ is the function $d_{A}: \mathbb{R}^{N} \rightarrow \mathbb{R}_{+}$given by $d_{A}(v)=\inf \{\|v-a\|: a \in A\}$. The function $d_{A}$ is globally Lipschitz with Lipschitz constant 1, that is, $\left\|d_{A}(v)-d_{A}(w)\right\| \leq\|v-w\|$ for all $v$ and $w$ in $\mathbb{R}^{N}$. A function $x: \mathbb{R}_{+} \rightarrow \mathbb{R}^{N}$ is said to approach the set $A$ if $d_{A}(x(t)) \rightarrow 0$ as $t \rightarrow \infty$. For $\varepsilon>0, \mathbb{B}_{\varepsilon}(A):=\left\{\xi \in \mathbb{R}^{N}: d_{A}(\xi)<\varepsilon\right\}$ (the $\varepsilon$-neighbourhood of $A$ ); for $a$ in $\mathbb{R}^{N}$, we write $\mathbb{B}_{\varepsilon}(a)$ in place of $\mathbb{B}_{\varepsilon}(\{a\})$. It is convenient to set $\mathbb{B}_{\varepsilon}(\emptyset)=\emptyset$. The closure of $A$ is denoted by $\operatorname{cl}(A)$. 
In his well-known book [4, p. 197], Birkhoff introduced the notion of an $\omega$-limit in the context of trajectories of dynamical systems. For the purposes of this paper, it is useful to define the concept of an $\omega$-limit point for arbitrary $\mathbb{R}^{N}$-valued functions defined on $\mathbb{R}_{+}$(see also [16, p. 112]). Let $x: \mathbb{R}_{+} \rightarrow \mathbb{R}^{N}$. A point $\xi$ of $\mathbb{R}^{N}$ is an $\omega$-limit point of $x$ if there exists an unbounded sequence $\left(t_{n}\right)$ in $\mathbb{R}_{+}$such that $x\left(t_{n}\right) \rightarrow \xi$ as $n \rightarrow \infty$; the (possibly empty) $\omega$-limit set of $x$, denoted by $\Omega(x)$, is the set of all $\omega$-limit points of $x$. The following well-known properties of $\omega$-limit sets (see, for example, [1], [16], and [31]) are summarized here for later reference (see also Figure 1).

Lemma 2.1. The following hold for any function $x: \mathbb{R}_{+} \rightarrow \mathbb{R}^{N}$ :

(a) $\Omega(x)$ is closed.

(b) $\Omega(x)=\emptyset$ if and only if $\|x(t)\| \rightarrow \infty$ as $t \rightarrow \infty$.

(c) If $x$ is continuous and bounded, then $\Omega(x)$ is nonempty, compact, and connected, is approached by $x$, and is the smallest closed set approached by $x$.

(d) If $x$ is continuous and $\Omega(x)$ is nonempty and bounded, then $x$ is bounded and $x$ approaches $\Omega(x)$.

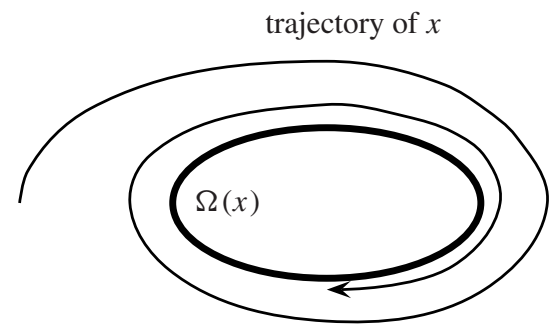

Figure 1.

\section{MOTIVATION: BARBĂLAT'S LEMMA, LASALLE'S INVARIANCE} PRINCIPLE, AND LYAPUNOV STABILITY. A function $y: \mathbb{R}_{+} \rightarrow \mathbb{R}$ is Riemann integrable (on $\mathbb{R}_{+}$) if the improper Riemann integral $\int_{0}^{\infty} y(s) d s$ exists, that is, $y$ is Riemann integrable on $[0, t]$ for each $t \geq 0$ and the $\operatorname{limit}_{t \rightarrow \infty} \int_{0}^{t} y(s) d s$ exists and is finite. If $y$ belongs to $L^{1}$ and is Riemann integrable on $[0, t]$ for each $t \geq 0$, then $y$ is Riemann integrable on $\mathbb{R}_{+}$. Furthermore, if $y$ is ultimately nonnegative (respectively, nonpositive) in the sense that, for some $\tau$ in $\mathbb{R}_{+}, y(t) \geq 0$ (respectively, $y(t) \leq 0$ ) whenever $t \geq \tau$, then Riemann integrability of $y$ implies that $y$ is in $L^{1}$. However, if $y$ is neither ultimately nonnegative nor ultimately nonpositive, the improper Riemann integral $y$ may exist, but $y$ may fail to be Lebesgue integrable.

As a starting point, we highlight the following simple observation, due to Barbălat [3]:

Lemma 3.1 (Barbălat's Lemma). If $y: \mathbb{R}_{+} \rightarrow \mathbb{R}$ is uniformly continuous and Riemann integrable, then $y(t) \rightarrow 0$ as $t \rightarrow \infty$.

Proof. Suppose to the contrary that $y(t) \not \rightarrow 0$ as $t \rightarrow \infty$. Then there exist $\varepsilon>0$ and a sequence $\left(t_{n}\right)$ in $\mathbb{R}_{+}$such that $t_{n} \rightarrow \infty$ as $n \rightarrow \infty$ and $\left|y\left(t_{n}\right)\right| \geq \varepsilon$ for all $n$ in $\mathbb{N}$. By the uniform continuity of $y$, there exists $\delta>0$ such that, for all $n$ in $\mathbb{N}$ and all $t$ in $\mathbb{R}_{+}$,

$$
\left|t_{n}-t\right| \leq \delta \quad \Longrightarrow \quad\left|y\left(t_{n}\right)-y(t)\right| \leq \varepsilon / 2
$$


Therefore, for all $t$ in $\left[t_{n}, t_{n}+\delta\right]$ and all $n$ in $\mathbb{N},|y(t)| \geq\left|y\left(t_{n}\right)\right|-\left|y\left(t_{n}\right)-y(t)\right| \geq \varepsilon / 2$, from which it follows that

$$
\left|\int_{0}^{t_{n}+\delta} y(t) d t-\int_{0}^{t_{n}} y(t) d t\right|=\left|\int_{t_{n}}^{t_{n}+\delta} y(t) d t\right|=\int_{t_{n}}^{t_{n}+\delta}|y(t)| d t \geq \frac{\varepsilon \delta}{2}>0
$$

for each $n$ in $\mathbb{N}$. By hypothesis, the improper Riemann integral $\int_{0}^{\infty} y(t) d t$ exists, and thus the left-hand side of the inequality converges to 0 as $n \rightarrow \infty$, yielding a contradiction.

Lemma 3.1 was originally derived in [3] to facilitate the analysis of the asymptotic behaviour of a class of systems of nonlinear second-order equations with forcing. Subsequently, Barbălat's lemma has been widely used in mathematical control theory (see, for example, [9, p. 89], [25, p. 211], and [28, p. 205]).

The following corollary is an immediate consequence of statement (c) of Lemma 2.1 and Lemma 3.1.

Corollary 3.2. Let $G$ be nonempty closed subset of $\mathbb{R}^{N}$, and let $g: G \rightarrow \mathbb{R}$ be continuous. Assume that $x: \mathbb{R}_{+} \rightarrow \mathbb{R}^{N}$ is bounded and uniformly continuous with $x\left(\mathbb{R}_{+}\right) \subset G$. If $g \circ x$ is Riemann integrable, then $\Omega(x) \subset g^{-1}(0)$ and $x$ approaches $g^{-1}(0)$.

Elaborating on the arguments sketched in section 1, we will use Corollary 3.2 to derive LaSalle's invariance principle. Let $f: \mathbb{R}^{N} \rightarrow \mathbb{R}^{N}$ be locally Lipschitz and consider the initial-value problem

$$
\dot{x}=f(x), \quad x(0)=x^{0} \in \mathbb{R}^{N} .
$$

Let $\varphi$ denote the corresponding local flow, that is, $t \mapsto \varphi\left(t, x^{0}\right)$ is the unique solution of (1) defined on $I\left(x^{0}\right)$, its maximal interval of existence. It is well known that, if $\mathbb{R}_{+} \subset I\left(x^{0}\right)$ and $\varphi\left(\cdot, x^{0}\right)$ is bounded on $\mathbb{R}_{+}$, then $\Omega\left(\varphi\left(\cdot, x^{0}\right)\right)$ is invariant with respect to the local flow $\varphi$ (see, for example, [1]).

The following "integral-invariance principle" is an easy consequence of Corollary 3.2.

Proposition 3.3. Let $G$ be a nonempty closed subset of $\mathbb{R}^{N}$, let $g: G \rightarrow \mathbb{R}$ be continuous, and let $x^{0}$ be a point of $G$. Assume that $\mathbb{R}_{+} \subset I\left(x^{0}\right), \varphi\left(\cdot, x^{0}\right)$ is bounded on $\mathbb{R}_{+}$, and $\varphi\left(\mathbb{R}_{+}, x^{0}\right) \subset G$. If the function $t \mapsto g\left(\varphi\left(t, x^{0}\right)\right)$ is Riemann integrable on $\mathbb{R}_{+}$, then $\varphi\left(\cdot, x^{0}\right)$ approaches the largest invariant subset contained in $g^{-1}(0)$.

Proof. Since $\varphi\left(\cdot, x^{0}\right)$ is bounded on $\mathbb{R}_{+}$and satisfies the differential equation, it follows that the derivative of $\varphi\left(\cdot, x^{0}\right)$ is bounded on $\mathbb{R}_{+}$. Consequently, $\varphi\left(\cdot, x^{0}\right)$ is uniformly continuous on $\mathbb{R}_{+}$. An application of Corollary 3.2 together with the invariance property of the $\omega$-limit set of $\varphi\left(\cdot, x^{0}\right)$ establishes the claim.

Proposition 3.3 is essentially contained in [5, Theorem 1.2]: the proof given therein is not based on Barbălat's lemma. The foregoing proof of Proposition 3.3 is from [11]. LaSalle's invariance principle (announced in [17], with proof in [18]) is a straightforward consequence of Proposition 3.3. For a continuously differentiable function $V: D \subset \mathbb{R}^{N} \rightarrow \mathbb{R}$ (where $D$ is open), it is convenient to define the directional derivative $V_{f}: D \rightarrow \mathbb{R}$ of $V$ in the direction of the vector field $f$ by $V_{f}(\xi)=\langle\nabla V(\xi), f(\xi)\rangle$. 
Corollary 3.4 (LaSalle's Invariance Principle). Let $D$ be a nonempty open subset of $\mathbb{R}^{N}$, let $V: D \rightarrow \mathbb{R}$ be continuously differentiable, and let $x^{0}$ be a point of $D$. Assume that $\mathbb{R}_{+} \subset I\left(x^{0}\right)$ and that there exists a compact subset $G$ of $\mathbb{R}^{N}$ such that $\varphi\left(\mathbb{R}_{+}, x^{0}\right) \subset G \subset D$. If $V_{f}(\xi) \leq 0$ for all $\xi$ in $G$, then $\varphi\left(\cdot, x^{0}\right)$ approaches the largest invariant subset contained in $V_{f}^{-1}(0) \cap G$.

Proof. By the compactness of $G$ and the continuity of $V$ on $G$, the function $V$ is bounded on $G$. Combining this with

$$
\int_{0}^{t} V_{f}\left(\varphi\left(s, x^{0}\right)\right) d s=\int_{0}^{t}(d / d s) V\left(\varphi\left(s, x^{0}\right)\right) d s=V\left(\varphi\left(t, x^{0}\right)\right)-V\left(x^{0}\right),
$$

we conclude that the function $t \mapsto \int_{0}^{t} V_{f}\left(\varphi\left(s, x^{0}\right)\right) d s$ is bounded from below. But this function is also nonincreasing (because $V_{f} \leq 0$ on $G$ ), hence the limit of $\int_{0}^{t} V_{f}\left(\varphi\left(s, x^{0}\right)\right) d s$ exists and is finite as $t \rightarrow \infty$, showing that the function $t \mapsto$ $V_{f}\left(\varphi\left(t, x^{0}\right)\right)$ is Riemann integrable on $\mathbb{R}_{+}$. An application of Proposition 3.3 (with $\left.g=\left.V_{f}\right|_{G}\right)$ completes the proof.

Assume that $f(0)=0$, that is, 0 is an equilibrium of (1). The equilibrium 0 is said to be stable if for every $\varepsilon>0$ there exists $\delta>0$ such that if $\left\|x^{0}\right\| \leq \delta$, then $\mathbb{R}_{+} \subset I\left(x^{0}\right)$ and $\left\|\varphi\left(t, x^{0}\right)\right\| \leq \varepsilon$ for all $t$ in $\mathbb{R}_{+}$. Furthermore, the equilibrium 0 is said to be asymptotically stable if it is stable and there exists $\delta>0$ such that $\left\|\varphi\left(t, x^{0}\right)\right\| \rightarrow 0$ as $t \rightarrow \infty$ for every $x^{0}$ satisfying $\left\|x^{0}\right\| \leq \delta$. We recall the following result due to Lyapunov (the proof of which is found, for example, in [13, p. 102], [16, pp. 154], and [31, p. 319]).

Theorem 3.5 (Lyapunov's Stability Theorem). Let D be a nonempty open subset of $\mathbb{R}^{N}$ such that 0 belongs to $D$, and let $V: D \rightarrow \mathbb{R}$ be continuously differentiable with $V(0)=0$. The following statements hold:

(a) If $V(\xi)>0$ for all $\xi$ in $D \backslash\{0\}$ and $V_{f}(\xi) \leq 0$ for all $\xi$ in $D$, then 0 is a stable equilibrium.

(b) If $V(\xi)>0$ and $V_{f}(\xi)<0$ for all $\xi$ in $D \backslash\{0\}$, then 0 is an asymptotically stable equilibrium.

Combining Corollary 3.4 and part (a) of Theorem 3.5, we immediately obtain the following generalization of part (b) of Theorem 3.5.

Corollary 3.6. Let $D$ be a nonempty open subset of $\mathbb{R}^{N}$ such that $0 \in D$, and let $V: D \rightarrow \mathbb{R}$ be continuously differentiable with $V(0)=0$. If $V(\xi)>0$ for all $\xi$ in $D \backslash\{0\}, V_{f}(\xi) \leq 0$ for all $\xi$ in $D$, and $\{0\}$ is the largest invariant subset of $V_{f}^{-1}(0)$, then 0 is an asymptotically stable equilibrium.

Corollary 3.6 frequently turns out to be useful in situations where the natural choice for $V$ does not satisfy the condition of strict negativity of $V_{f}$ required in part (b) of Theorem 3.5.

Example 3.7. In this example, we describe a typical application of Corollary 3.6 in the context of a general class of nonlinear second-order systems. Consider the system

$$
\ddot{y}(t)+r(y(t), \dot{y}(t))=0, \quad(y(0), \dot{y}(0))=\left(p^{0}, v^{0}\right) \in \mathbb{R}^{2},
$$


where $r: \mathbb{R}^{2} \rightarrow \mathbb{R}$ is locally Lipschitz and continuously differentiable with respect to the second variable. Furthermore, we assume that $r(0,0)=0$. Setting $x(t)=\left(x_{1}(t), x_{2}(t)\right)=(y(t), \dot{y}(t))$, the second-order system (2) can be expressed in the equivalent form (1), where $f: \mathbb{R}^{2} \rightarrow \mathbb{R}^{2}$ and $x^{0}$ in $\mathbb{R}^{2}$ are given by

$$
f(p, v)=(v,-r(p, v)), \quad x^{0}=\left(p^{0}, v^{0}\right) .
$$

Let $\varepsilon>0$, set $D=(-\varepsilon, \varepsilon) \times(-\varepsilon, \varepsilon)$, and define $V: D \rightarrow \mathbb{R}$ by

$$
(p, v) \mapsto \int_{0}^{p} r(s, 0) d s+v^{2} / 2
$$

It follows from the mean-value theorem that, for each $(p, v)$ in $D$, there exists a number $\theta=\theta(p, v)$ in the interval $(0,1)$ such that

$$
V_{f}(p, v)=-v(r(p, v)-r(p, 0))=-v^{2} \frac{\partial r}{\partial v}(p, \theta v) .
$$

Claim. Consider (1) with $f$ and $x^{0}$ given by (3). If $\operatorname{pr}(p, 0)>0$ for all $p$ in $(-\varepsilon, \varepsilon) \backslash\{0\}$ and $(\partial r / \partial v)(p, v)>0$ for all $(p, v)$ in $D$ satisfying $p v \neq 0$, then the equilibrium 0 is asymptotically stable.

We proceed to establish this claim. Using the hypotheses and (4), we infer that $V(p, v)>0$ for all $(p, v)$ in $D \backslash\{0\}$ and $V_{f}(p, v) \leq 0$ for all $(p, v)$ in $D$. Observe that $V_{f}^{-1}(0)$ is contained in $\{(p, v) \in D: p v=0\}$ and contains $\{(p, v) \in D: v=0\}$ (implying in particular that the claim does not follow from part (b) of Theorem 3.5). Writing $\varphi\left(t, x^{0}\right)=\left(x_{1}(t), x_{2}(t)\right)$, we see that for $x^{0}=\left(p^{0}, 0\right)$ in $D$ with $p^{0} \neq 0$, $\dot{x}_{2}(0)=-r\left(p^{0}, 0\right) \neq 0$. Similarly, for $x^{0}=\left(0, v^{0}\right)$ in $D$ with $v^{0} \neq 0, \dot{x}_{1}(0)=v^{0} \neq 0$. We conclude that solutions with these initial conditions do not remain in $V_{f}^{-1}(0)$, showing that $\{0\}$ is the largest invariant subset of $V_{f}^{-1}(0)$. The claim now follows from Corollary 3.6.

As a special case of (2), we consider the Liénard equation

$$
\ddot{y}(t)+d(y(t)) \dot{y}(t)+k(y(t))=0, \quad(y(0), \dot{y}(0))=\left(p^{0}, v^{0}\right) \in \mathbb{R}^{2},
$$

which describes a nonlinear oscillator, where $d(y) \dot{y}$ represents a friction term that is linear in the velocity and $k(y)$ models a restoring force. We assume that the functions $d: \mathbb{R} \rightarrow \mathbb{R}$ and $k: \mathbb{R} \rightarrow \mathbb{R}$ are locally Lipschitz and $k(0)=0$. It follows from the foregoing discussion on the stability behaviour of (2) (applied to $r$ given by $r(p, v)=$ $d(p) v+k(p))$ that 0 is an asymptotically stable equilibrium state of the Liénard equation, provided that there exists $\varepsilon>0$ such that $p k(p)>0$ and $d(p)>0$ for all $p$ in $(-\varepsilon, \varepsilon)$ with $p \neq 0$.

We mention that there are many situations of interest in control theory where the integrability condition in Proposition 3.3 is automatically satisfied, for example, in optimal control (finiteness of an integral performance criterion). Proposition 3.3 is particularly useful in the context of observed systems. In applications, it is frequently impossible to observe or measure the complete state $x(t)$ of (1) at time $t$. To illustrate the latter comment, consider the observed system given by (1) and the observation

$$
z=c(x),
$$

where $c: \mathbb{R}^{N} \rightarrow \mathbb{R}^{P}$ is continuous with $c(0)=0$ (see Figure 2 for a schematic illustration). The observation $z$ (also called output or measurement) depends on the state 
and should be thought of as a quantity that can be observed or measured, an important special case occurring when $z$ is given by one component of the state. Observability concepts relate to the issue of precluding different initial states generating the same observation: the initial state of an observable system can in principle be recovered from the observation. The system given by (1) and (5) is said to be zero-state observable if the following holds for each $x^{0}$ in $\mathbb{R}^{N}$ :

$$
z(\cdot)=c\left(\varphi\left(\cdot, x^{0}\right)\right)=0 \quad \Longrightarrow \quad \varphi\left(\cdot, x^{0}\right)=0,
$$

that is, the system is zero-state observable if $x(\cdot)=0$ is the only solution generating the zero observation $z(\cdot)=0$. As an example consider system (2) introduced in Example 3.7. Endowed with the observation $z=x_{1}=y$ (that is, $c(p, v)=p$ ), it is trivial that the resulting observed system is zero-state observable. Similarly, it is immediate that, with the observation $z=x_{2}=\dot{y}$ (that is, $c(p, v)=v$ ), the system is zero-state observable if and only if $r(p, 0) \neq 0$ for all $p \neq 0$.

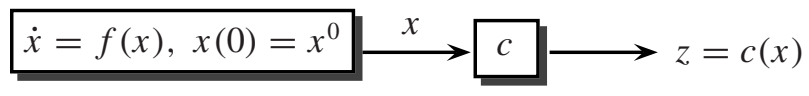

Figure 2.

The following corollary of Proposition 3.3 is contained in [5, Theorem 1.3] and essentially states that, for a zero-state observable system, every bounded trajectory with observation in $L^{p}$ necessarily converges to zero.

Corollary 3.8. Assume that the observed system given by (1) and (5) is zero-state observable. For given $x^{0}$ in $\mathbb{R}^{N}$ assume that $\mathbb{R}_{+} \subset I\left(x^{0}\right)$ and that $\varphi\left(\cdot, x^{0}\right)$ is bounded on $\mathbb{R}_{+}$. If $\int_{0}^{\infty}\left\|c\left(\varphi\left(t, x^{0}\right)\right)\right\|^{p} d t<\infty$ for some $p$ in $(0, \infty)$, then $\lim _{t \rightarrow \infty} \varphi\left(t, x^{0}\right)=0$.

Proof. By the continuity and boundedness of $\varphi\left(\cdot, x^{0}\right)$, it follows from Lemma 2.1 that $\varphi\left(\cdot, x^{0}\right)$ approaches its $\omega$-limit set $\Omega:=\Omega\left(\varphi\left(\cdot, x^{0}\right)\right)$ and that $\Omega$ is the smallest closed set approached by $\varphi\left(\cdot, x^{0}\right)$. An application of Proposition 3.3 with $G=\mathbb{R}^{N}$ and $g(\cdot)=\|c(\cdot)\|^{p}$ shows that $\Omega \subset g^{-1}(0)=c^{-1}(0)$. Let $\xi$ be a point of $\Omega$. By the invariance property of $\Omega, \varphi(t, \xi)$ lies in $\Omega$ for all $t$ in $\mathbb{R}$. Consequently, $c(\varphi(\cdot, \xi))=0$. Zero-state observability ensures that $\varphi(\cdot, \xi)=0$, showing that $\xi=0$. Hence $\Omega=\{0\}$, so $\lim _{t \rightarrow \infty} \varphi\left(t, x^{0}\right)=0$.

4. GENERALIZATIONS OF BARBĂLAT'S LEMMA. In Theorems 4.4 and 4.5, we present generalizations of Barbălat's lemma and of Corollary 3.2 that allow interesting applications to differential equations. To this end we introduce the concept of (weak) meagreness that will replace the assumption of Riemann integrability in Barbălat's lemma. Recall that $\mu$ denotes Lebesgue measure on $\mathbb{R}_{+}$.

\section{Definition 4.1.}

(a) A function $y: \mathbb{R}_{+} \rightarrow \mathbb{R}$ is said to be meagre if $y$ is Lebesgue measurable and $\mu\left(\left\{t \in \mathbb{R}_{+}:|y(t)| \geq \lambda\right\}\right)<\infty$ for all $\lambda>0$.

(b) A function $y: \mathbb{R}_{+} \rightarrow \mathbb{R}$ is said to be weakly meagre if $\lim _{n \rightarrow \infty}\left(\inf _{t \in I_{n}}|y(t)|\right)=$ 0 for every family $\left\{I_{n}: n \in \mathbb{N}\right\}$ of nonempty and pairwise disjoint closed intervals $I_{n}$ in $\mathbb{R}_{+}$with $\inf _{n \in \mathbb{N}} \mu\left(I_{n}\right)>0$. 
We remark that, in the theory of rearrangements of functions, the property of meagreness is sometimes referred to as "vanishing at infinity" (see [21, p. 72]). Moreover, it is easy to link meagreness to the well-known concept of convergence in measure (see, for example, [14]). To do this, let $y: \mathbb{R}_{+} \rightarrow \mathbb{R}$ be Lebesgue measurable. For each $n$ in $\mathbb{N}$ define a function $y_{n}: \mathbb{R}_{+} \rightarrow \mathbb{R}$ by

$$
y_{n}(t)= \begin{cases}0 & \text { if } t \in[0, n], \\ y(t) & \text { if } t>n\end{cases}
$$

Then it is a routine exercise to show that $y$ is meagre if and only if

$$
\lim _{n \rightarrow \infty} \mu\left(\left\{t \in \mathbb{R}_{+}:\left|y_{n}(t)\right|>\varepsilon\right\}\right)=0
$$

for each $\varepsilon>0$, that is, if and only if $y_{n}$ converges to 0 in measure as $n \rightarrow \infty$.

From Definition 4.1 it follows immediately that a meagre function is weakly meagre. The converse is not true, even in the restricted context of continuous functions (see Example 7.1 in the appendix). It is clear that if a function $y: \mathbb{R}_{+} \rightarrow \mathbb{R}$ is weakly meagre, then 0 belongs to $\Omega(y)$.

The following result gives sufficient conditions for meagreness and weak meagreness, respectively.

Proposition 4.2. Let $y: \mathbb{R}_{+} \rightarrow \mathbb{R}$ be measurable. Then the following statements hold:

(a) If there exists a Borel function $\alpha: \mathbb{R}_{+} \rightarrow \mathbb{R}$ such that

$$
\alpha^{-1}(0)=\{0\}, \quad \inf _{s \geq \sigma} \alpha(s)>0
$$

for all $\sigma>0$, and $\alpha(|y(\cdot)|)$ belongs to $L^{1}$, then $y$ is meagre.

(b) If there exists $\tau>0$ such that $\int_{t}^{t+\tau}|y(s)| d s$ converges to 0 as $t \rightarrow \infty$, then $y$ is weakly meagre.

(c) If $y$ is continuous and for every $\delta>0$ there exists $\tau$ in $(0, \delta)$ such that $\int_{t}^{t+\tau} y(s) d s$ converges to 0 as $t \rightarrow \infty$, then $y$ is weakly meagre.

Proof. We prove only part (c) (the proofs of parts (a) and (b) are even more straightforward). Let $y: \mathbb{R}_{+} \rightarrow \mathbb{R}$ be continuous. We show that if $y$ is not weakly meagre, then there exists $\delta>0$ such that for every $\tau$ in $(0, \delta)$ the integral $\int_{t}^{t+\tau} y(s) d s$ does not converge to 0 as $t \rightarrow \infty$. The claim follows then from contraposition. So assume that $y$ is not weakly meagre. Then there exists a family $\left\{I_{n}: n \in \mathbb{N}\right\}$ of nonempty, pairwise disjoint closed intervals with $\delta=\inf _{n \in \mathbb{N}} \mu\left(I_{n}\right)>0$ and a number $\varepsilon>0$ such that $\inf _{t \in I_{n}}|y(t)| \geq \varepsilon$ for each $n$. Since $y$ is continuous, the function $y$ has the same sign on $I_{n}$ for each $n$. Without loss of generality, we may assume that there are infinitely many intervals $I_{n}$ on which $y$ is positive. Then there exists a sequence $\left(n_{k}\right)$ in $\mathbb{N}$ such that $y$ has positive sign on $I_{n_{k}}$ for all $k$. Denoting the left endpoint of $I_{n_{k}}$ by $t_{k}$, we obtain

$$
\int_{t_{k}}^{t_{k}+\tau} y(s) d s \geq \varepsilon \tau>0
$$

for each $k$ in $\mathbb{N}$ and $\tau$ in $(0, \delta)$, showing that the integral $\int_{t}^{t+\tau} y(s) d s$ does not converge to 0 as $t \rightarrow \infty$. 
It follows immediately from Proposition 4.2(a) that, if $y$ belongs to $L^{p}$ for some $p$ in $(0, \infty)$, then $y$ is meagre. Part (c) shows, in particular, that if $y: \mathbb{R}_{+} \rightarrow \mathbb{R}$ is continuous and Riemann integrable on $\mathbb{R}_{+}$, then $y$ is weakly meagre. However, we mention that continuity and Riemann integrability of a function $y: \mathbb{R}_{+} \rightarrow \mathbb{R}$ do not guarantee that $y$ is meagre (Example 7.1 in the appendix describes a nonmeagre function that is both continuous and Riemann integrable). The sufficient conditions for weak meagreness provided by parts (b) and (c) of Proposition 4.2 are not necessary. To illustrate this, we construct in Example 7.2 a continuous function $y$ that is meagre (and so a fortiori is weakly meagre), but is such that, for each $\tau>0$, the integral $\int_{t}^{t+\tau} y(s) d s$ does not converge to 0 as $t \rightarrow \infty$. By contrast, the sufficient condition for meagreness given in Proposition 4.2(a) is also necessary (for a proof of this assertion, see [22]).

The following result will play a role in the subsequent derivation of generalized versions of Barbălat's lemma.

Lemma 4.3. Let $A$ and $B$ be nonempty subsets of $\mathbb{R}^{N}$ such that $\operatorname{cl}\left(\mathbb{B}_{\lambda}(B)\right) \subset A$ for some $\lambda>0$. If $x: \mathbb{R}_{+} \rightarrow \mathbb{R}^{N}$ is uniformly continuous on $x^{-1}(A)$, then there exists $\tau>0$ such that

$$
t \in \mathbb{R}_{+}, x(t) \in B \quad \Longrightarrow \quad x(s) \in \mathbb{B}_{\lambda}(B) \quad\left(s \in[t-\tau, t+\tau] \cap \mathbb{R}_{+}\right) .
$$

Proof. Seeking a contradiction, we suppose that property (6) does not hold. Then there exist sequences $\left(s_{n}\right)$ and $\left(t_{n}\right)$ in $\mathbb{R}_{+}$such that $x\left(t_{n}\right) \in B$ and $x\left(s_{n}\right) \notin \mathbb{B}_{\lambda}(B)$ for all $n$, and $s_{n}-t_{n} \rightarrow 0$ as $n \rightarrow \infty$. Evidently, $s_{n} \neq t_{n}$ for all $n$. Define $I_{n}$ to be the closed interval with left endpoint $\min \left\{s_{n}, t_{n}\right\}$ and right endpoint $\max \left\{s_{n}, t_{n}\right\}$, and write $T_{n}=\left\{s \in I_{n}: s \notin x^{-1}\left(\mathbb{B}_{\lambda}(B)\right)\right\}$. For each $n$, let $\tau_{n}$ in $T_{n}$ (a compact set) be such that

$$
\left|\tau_{n}-t_{n}\right|=\min _{s \in T_{n}}\left|s-t_{n}\right|
$$

Clearly, $d_{B}\left(x\left(\tau_{n}\right)\right)=\lambda$ and $d_{B}\left(x\left(t_{n}\right)\right)=0$ for each $n$. Combining this information with the facts that $\tau_{n}$ belongs to $I_{n}$ and $\lim _{n \rightarrow \infty}\left(s_{n}-t_{n}\right)=0$, we conclude that

(i) $\left\|x\left(t_{n}\right)-x\left(\tau_{n}\right)\right\| \geq\left|d_{B}\left(x\left(t_{n}\right)\right)-d_{B}\left(x\left(\tau_{n}\right)\right)\right|=\lambda>0$,

(ii) $t_{n}, \tau_{n} \in x^{-1}(A)$,

(iii) $\left|t_{n}-\tau_{n}\right| \rightarrow 0$ as $n \rightarrow \infty$,

contradicting the hypothesis of the uniform continuity of $x$ on $x^{-1}(A)$. Therefore, property (6) holds.

The following two theorems, the main results of this section, provide our generalizations of Barbălat's lemma.

Theorem 4.4. Let $G$ be a nonempty closed subset of $\mathbb{R}^{N}$, let $g: G \rightarrow \mathbb{R}$ be a function, and let $x: \mathbb{R}_{+} \rightarrow \mathbb{R}^{N}$ be continuous with $x\left(\mathbb{R}_{+}\right) \subset G$. Assume that each $\xi$ in $G$ for which $g(\xi) \neq 0$ has a neighbourhood $U$ such that

$$
\inf \{|g(w)|: w \in G \cap U\}>0
$$

and $x$ is uniformly continuous on $x^{-1}(U)$. If $g \circ x$ is weakly meagre, then the following statements hold:

(a) $\Omega(x)$ is contained in $g^{-1}(0)$. 
(b) If $g^{-1}(0)$ is bounded and $\Omega(x) \neq \emptyset$, then $x$ is bounded and $x$ approaches $g^{-1}(0)$.

(c) If $x$ is bounded, then $g^{-1}(0) \neq \varnothing$ and $x$ approaches $g^{-1}(0)$.

(d) If $x$ is bounded and $g^{-1}(0)$ is totally disconnected, then $\Omega(x)$ consists of a single point $x^{\infty}$ that lies in $g^{-1}(0)$ (in particular, $\left.\lim _{t \rightarrow \infty} x(t)=x^{\infty}\right)$.

Proof. If $\Omega(x)=\emptyset$, then statement (a) holds trivially. Now assume that $\Omega(x) \neq \emptyset$. Let $\xi$ be a point of $\Omega(x)$. Since $G$ is closed and $x\left(\mathbb{R}_{+}\right) \subset G, \Omega(x) \subset G$ and thus $\xi$ belongs to $G$. We show that $g(\xi)=0$. Seeking a contradiction, suppose that $g(\xi) \neq 0$. By the hypotheses, there exists a neighbourhood $U$ of $\xi$ such that (7) holds and $x$ is uniformly continuous on $x^{-1}(U)$. Choose $\delta>0$ such that the closure of $\mathbb{B}_{\delta}(\xi)$ lies in $U$. Then

$$
\varepsilon=\inf \left\{|g(w)|: w \in G \cap \mathbb{B}_{\delta}(\xi)\right\}>0 .
$$

Choose $\delta_{1}$ in $(0, \delta)$. Since $\xi$ is an element of $\Omega(x)$, there exists a sequence $\left(t_{n}\right)$ in $\mathbb{R}_{+}$ with $t_{n+1}-t_{n}>1$ and $x\left(t_{n}\right)$ in $\mathbb{B}_{\delta_{1}}(\xi)$ for all $n$. An application of Lemma 4.3 (with $A=U, B=\mathbb{B}_{\delta_{1}}(\xi)$, and $\left.\lambda=\delta-\delta_{1}\right)$ shows that there exists $\tau$ in $(0,1)$ such that $x(t)$ is in $\mathbb{B}_{\delta_{2}}(\xi)$ for all $t$ in $\cup_{n \in \mathbb{N}}\left[t_{n}, t_{n}+\tau\right]$. Therefore, by (8),

$$
|(g \circ x)(t)| \geq \varepsilon \quad\left(t \in\left[t_{n}, t_{n}+\tau\right], n \in \mathbb{N}\right) .
$$

Finally, since $t_{n+1}-t_{n}>1$ for all $n$ and $\tau$ belongs to $(0,1)$, the intervals $\left[t_{n}, t_{n}+\tau\right]$ are pairwise disjoint. Combined with (9) this contradicts the weak meagreness of $g \circ x$ and establishes (a).

A combination of statement (a) and Lemma 2.1 yields statements (b)-(d).

We remark that lower semicontinuity of the function $\xi \mapsto|g(\xi)|$ is sufficient to ensure that (7) holds for some neighbourhood $U$ of any $\xi$ in $G$ with $g(\xi) \neq 0$.

Barbălat's lemma follows immediately from an application of Theorem 4.4(b) to the situation wherein $N=1, G=\mathbb{R}, g=\mathrm{id}_{\mathbb{R}}$, and $x=y$, in conjunction with the observation that a uniformly continuous and Riemann integrable function $y: \mathbb{R}_{+} \rightarrow \mathbb{R}$ is weakly meagre, implying that 0 is a member of $\Omega(y)$ and thus ensuring that $\Omega(y) \neq \emptyset$. Corollary 3.2 is a simple consequence of statements (a) and (c) of Theorem 4.4.

When compared with Theorem 4.4, the next result (Theorem 4.5) posits that $x$ be uniformly continuous on $x^{-1}\left(\mathbb{B}_{\varepsilon}\left(g^{-1}(0)\right)\right)$ for some $\varepsilon>0$. We remark that, in certain situations (for example, if $g^{-1}(0)$ is finite), this assumption is weaker than the uniform continuity assumption imposed on $x$ in Theorem 4.4. On the other hand, the assumption imposed on $g$ in Theorem 4.5 is stronger than its counterpart in Theorem 4.4. However, under these modified hypotheses, Theorem 4.5 guarantees that $x$ approaches $g^{-1}(0) \neq \varnothing$ without assuming the nonemptiness of $\Omega(x)$ or the boundedness of $x$.

Theorem 4.5. Let $G$ be a nonempty closed subset of $\mathbb{R}^{N}$, and let $g: G \rightarrow \mathbb{R}$ be such that $g^{-1}(0)$ is closed and such that, for every nonempty closed subset $K$ of $G$,

$$
\inf _{\xi \in K}|g(\xi)|>0
$$

whenever $K \cap g^{-1}(0)=\emptyset$. Furthermore, let $x: \mathbb{R}_{+} \rightarrow \mathbb{R}^{N}$ be continuous with $x\left(\mathbb{R}_{+}\right) \subset G$. If (i) $x$ is uniformly continuous on $x^{-1}\left(\mathbb{B}_{\varepsilon}\left(g^{-1}(0)\right)\right)$ for some $\varepsilon>0$ and (ii) $g \circ x$ is weakly meagre, then the following statements hold: 
(a) $g^{-1}(0) \neq \emptyset$, $x$ approaches $g^{-1}(0)$, and $\Omega(x)$ is contained in $g^{-1}(0)$.

(b) If $g^{-1}(0)$ is bounded, then $x$ is bounded, $x$ approaches $g^{-1}(0)$, and $\Omega(x)$ is a nonempty subset of $g^{-1}(0)$.

(c) If $g^{-1}(0)$ is bounded and totally disconnected, then $\Omega(x)$ is a singleton $\left\{x^{\infty}\right\}$, where $x^{\infty}$ is a point of $g^{-1}(0)$ (hence, $\left.\lim _{t \rightarrow \infty} x(t)=x^{\infty}\right)$.

Proof. For convenience, we set $Z=g^{-1}(0)$. It is clear that $Z \neq \varnothing$ (otherwise, by (10) and the closedness of $G, \gamma=\inf _{\xi \in G}|g(\xi)|>0$ and so $|g(x(t))| \geq \gamma$ for all $t$ in $\mathbb{R}_{+}$, which contradicts the weak meagreness of $g \circ x$ ). To prove statements (a) and (b), it now suffices to show that $x$ approaches $Z$. From the closedness of $Z$ it then follows immediately that $\Omega(x) \subset Z$; moreover, if $Z$ is bounded, then we can conclude that $x$ is bounded and so $\Omega(x) \neq \emptyset$. Since, by assumption, the trajectory of $x$ is contained in $G$, it is immediate that, if $G=Z$, then $x$ approaches $Z$. Consider the remaining case, wherein $Z$ is a proper subset of $G$. By the closedness of $Z$, there exists $\delta$ in $(0, \varepsilon / 3)$ such that $G \backslash \mathbb{B}_{\delta}(Z) \neq \emptyset$. For $\theta$ in $(0, \delta)$, define

$$
\iota(\theta)=\inf \left\{|g(\xi)|: \xi \in G \backslash \mathbb{B}_{\theta}(Z)\right\}>0,
$$

wherein positivity is a consequence of (10) and the closedness of $G \backslash \mathbb{B}_{\theta}(Z)$.

In search of a contradiction, we suppose that $\lim _{t \rightarrow \infty} d_{Z}(x(t)) \neq 0$. Then there exist $\lambda$ in $(0, \delta)$ and a sequence $\left(t_{n}\right)$ in $\mathbb{R}_{+}$with $t_{n} \rightarrow \infty$ as $n \rightarrow \infty$ and $d_{Z}\left(x\left(t_{n}\right)\right) \geq 3 \lambda$ for all $n$. By the weak meagreness of $g \circ x$, there exists a sequence $\left(s_{n}\right)$ in $\mathbb{R}_{+}$with $s_{n} \rightarrow \infty$ as $n \rightarrow \infty$ and $\left|g\left(x\left(s_{n}\right)\right)\right|<\iota(\lambda)$ for all $n$, so $d_{Z}\left(x\left(s_{n}\right)\right) \leq \lambda$ for all $n$. Extracting subsequences of $\left(t_{n}\right)$ and $\left(s_{n}\right)$ (which we do not relabel), we may assume that $s_{n}$ is in $\left(t_{n}, t_{n+1}\right)$ for all $n$. We now have

$$
d_{Z}\left(x\left(t_{n}\right)\right) \geq 3 \lambda, \quad d_{Z}\left(x\left(s_{n}\right)\right) \leq \lambda, \quad s_{n} \in\left(t_{n}, t_{n+1}\right)
$$

for all $n$. By the continuity of $d_{Z} \circ x$, there exists for each $n$ a number $\sigma_{n}$ in $\left(t_{n}, s_{n}\right)$ such that $x\left(\sigma_{n}\right)$ belongs to $B:=\left\{\xi \in G: d_{Z}(\xi)=2 \lambda\right\}$. Extracting a subsequence (which, again, we do not relabel), we may assume that $\sigma_{n+1}-\sigma_{n}>1$ for all $n$. Noting that $\operatorname{cl}\left(\mathbb{B}_{\lambda}(B)\right) \subset \mathbb{B}_{\varepsilon}(Z)$ and invoking Lemma 4.3 (with $A=\mathbb{B}_{\varepsilon}(Z)$ ), we conclude the existence of $\tau$ in $(0,1)$ such that $d_{Z}(x(t)) \geq \lambda$ for all $t$ in $\left[\sigma_{n}, \sigma_{n}+\tau\right]$ and all $n$. Therefore,

$$
\left\{t \in \mathbb{R}_{+}:|g(x(t))| \geq \iota(\lambda)\right\} \supset \cup_{n \in \mathbb{N}}\left[\sigma_{n}, \sigma_{n}+\tau\right]
$$

which (on noting that the intervals $\left[\sigma_{n}, \sigma_{n}+\tau\right]$ are each of length $\tau>0$ and form a pairwise disjoint family) contradicts the weak meagreness of $g \circ x$. Therefore, $x$ approaches $Z$, implying that statements (a) and (b) hold. Finally, invoking the fact that the $\omega$-limit set of a bounded continuous function is connected, we infer statement (c) from statement (b).

We mention that Barbălat's lemma follows immediately from Theorem 4.5(a).

5. APPLICATIONS TO NONAUTONOMOUS DIFFERENTIAL EQUATIONS. Consider the initial-value problem for a nonautonomous ordinary differential equation:

$$
\dot{x}(t)=f(t, x(t)), \quad x(0)=x^{0} \in \mathbb{R}^{N} .
$$


Throughout, $f: \mathbb{R}_{+} \times \mathbb{R}^{N} \rightarrow \mathbb{R}^{N}$ is a Carathéodory function. This means that: $f(\cdot, \xi)$ is Lebesgue measurable for each $\xi$ in $\mathbb{R}^{N} ; f(t, \cdot)$ is continuous for each $t$ in $\mathbb{R}_{+} ; f$ is locally integrably bounded on compact sets (i.e., for each compact subset $K$ of $\mathbb{R}^{N}$ there exists $m$ in $L_{\text {loc }}^{1}$ such that $\|f(t, \xi)\| \leq m(t)$ for all $(t, \xi)$ in $\left.\mathbb{R}_{+} \times K\right)$. For each $x^{0}$ in $\mathbb{R}^{N}$, there exists $\omega>0$ such that (11) has a solution on $[0, \omega)$, meaning a locally absolutely continuous function $x:[0, \omega) \rightarrow \mathbb{R}^{N}$ with $x(0)=x^{0}$ that satisfies the differential equation in (11) for almost all $t$ in $[0, \omega)$. Recall that a function is locally absolutely continuous if and only if it is a primitive of a locally integrable function. A solution of (11) on $\mathbb{R}_{+}$is said to be a global solution. A solution $x$ on $I=[0, \omega)$ is maximal (with maximal interval of existence $I$ ) if, for every $\tilde{\omega}>\omega$ and every solution $\tilde{x}$ of $(11)$ on $[0, \tilde{\omega})$, there exists $\tilde{t}$ in $(0, \omega)$ such that $\tilde{x}(\tilde{t}) \neq x(\tilde{t})$ (equivalently, the solution $x$ is maximal if it has no proper right extension that is also a solution). Every solution $x$ of (11) can be extended to a maximal solution on a maximal interval henceforth denoted by $\left[0, \omega_{x}\right)$; moreover, if $x$ is maximal and $\omega_{x}<\infty$, then $x$ is unbounded (see [8, Theorem 1.3, p. 47]). It follows that if a maximal solution is bounded, then it is global.

Definition 5.1. A function $m: \mathbb{R}_{+} \rightarrow \mathbb{R}$ is uniformly locally integrable if $m$ belongs to $L_{\text {loc }}^{1}$ and if for each $\varepsilon>0$ there exists $\tau>0$ such that

$$
\int_{t}^{t+\tau}|m(s)| d s \leq \varepsilon
$$

for all $t$ in $\mathbb{R}_{+}$.

Clearly, a locally integrable function $m: \mathbb{R}_{+} \rightarrow \mathbb{R}$ is uniformly locally integrable if and only if the function $t \mapsto \int_{0}^{t}|m(s)| d s$ is uniformly continuous. It is readily verified that, if $m$ belongs to $L^{p}$ for some $p(1 \leq p \leq \infty)$, then $m$ is uniformly locally integrable.

Definition 5.2. For a nonempty subset $A$ of $\mathbb{R}^{N}, \mathcal{F}(A)$ denotes the class of Carathéodory functions $f: \mathbb{R}_{+} \times \mathbb{R}^{N} \rightarrow \mathbb{R}^{N}$ with the property that there exists a uniformly locally integrable function $m$ such that $\|f(t, \xi)\| \leq m(t)$ for all $(t, \xi)$ in $\mathbb{R}_{+} \times A$.

The following proposition shows that under suitable uniform local integrability assumptions relating to $f$, solutions of (11) satisfy the uniform continuity assumptions required for an application of Theorems 4.4 and 4.5 .

Proposition 5.3. Let $A$ and $B$ be nonempty subsets of $\mathbb{R}^{N}$ with the property that $\mathbb{B}_{\varepsilon}(A) \cap B \neq \emptyset$ for some $\varepsilon>0$, and let $f$ belong to $\mathcal{F}\left(\mathbb{B}_{\varepsilon}(A) \cap B\right)$. If $x: \mathbb{R}_{+} \rightarrow \mathbb{R}^{N}$ is a global solution of (11) such that $x\left(\mathbb{R}_{+}\right) \subset B$, then $x$ is uniformly continuous on $x^{-1}(A)$.

Proof. If $x^{-1}(A)=\emptyset$, then the claim holds trivially. Assume that $x^{-1}(A) \neq \emptyset$. Since $f$ belongs to $\mathcal{F}\left(\mathbb{B}_{\varepsilon}(A) \cap B\right)$, there exists a uniformly locally integrable function $m$ such that $\|f(t, w)\| \leq m(t)$ for all $(t, w)$ in $\mathbb{R}_{+} \times\left(\mathbb{B}_{\varepsilon}(A) \cap B\right)$. Let $\delta$ in $(0, \varepsilon)$ be arbitrary. Choose $\tau>0$ such that $\int_{t}^{t+\tau} m \leq \delta$ for all $t$ in $\mathbb{R}_{+}$. Let $t_{1}$ and $t_{2}$ be points of $x^{-1}(A)$ with $0 \leq t_{2}-t_{1} \leq \tau$. We will complete the proof by showing that $\left\|x\left(t_{2}\right)-x\left(t_{1}\right)\right\| \leq \delta$. If we define

$$
J=\left\{t>t_{1}: x(s) \in \mathbb{B}_{\varepsilon}(A) \quad \text { for all } s \in\left[t_{1}, t\right]\right\}
$$


it follows that

$$
\left\|x(t)-x\left(t_{1}\right)\right\| \leq \int_{t_{1}}^{t} m(s) d s \leq \int_{t_{1}}^{t_{1}+\tau} m(s) d s \leq \delta
$$

for all $t$ in $J$ with $t \leq t_{1}+\tau$. Since $\delta<\varepsilon, t_{1}+\tau$ belongs to $J$, which ensures that $\left\|x\left(t_{2}\right)-x\left(t_{1}\right)\right\| \leq \delta$.

In the following, we combine Proposition 5.3 with Theorems 4.4 and 4.5 to derive results on the asymptotic behaviour of solutions of (11).

Theorem 5.4. Let $G$ be a nonempty closed subset of $\mathbb{R}^{N}$, and let $g: G \rightarrow \mathbb{R}$ be a function. Assume that each $\xi$ in $G$ for which $g(\xi) \neq 0$ has a neighbourhood $U$ such that (7) holds and $f$ belongs to $\mathcal{F}(U \cap G)$. If $x: \mathbb{R}_{+} \rightarrow \mathbb{R}^{N}$ is a global solution of (11) with $x\left(\mathbb{R}_{+}\right) \subset G$ and $g \circ x$ is weakly meagre, then statements (a)-(d) of Theorem 4.4 hold.

Proof. Let $\xi$ in $G$ be such that $g(\xi) \neq 0$. By the hypotheses, there exists a neighbourhood $U$ of $\xi$ such that (7) holds and $f$ belongs to $\mathcal{F}(U \cap G)$. Let $\varepsilon>0$ be sufficiently small that $\mathbb{B}_{2 \varepsilon}(\xi)$ lies in $U$. Then, setting $A=\mathbb{B}_{\varepsilon}(\xi)$, we see that $f$ is in the class $\mathcal{F}\left(\mathbb{B}_{\varepsilon}(A) \cap G\right)$. By Proposition 5.3, it follows that $x$ is uniformly continuous on $x^{-1}(A)$. An application of Theorem 4.4 completes the proof.

We remark that Theorem 5.4 contains a recent result by Teel [30, Theorem 1] as a special case. In the next theorem, it is assumed that $f$ is a member of

$$
\mathcal{F}\left(\mathbb{B}_{\varepsilon}\left(g^{-1}(0)\right) \cap G\right)
$$

for some $\varepsilon>0$. Under the additional assumption that $g$ satisfies (10), it is then guaranteed that $x$ approaches $g^{-1}(0)$ (without positing the boundedness of $x$ ).

Theorem 5.5. Let $G$ be a nonempty closed subset of $\mathbb{R}^{N}$, and let $g: G \rightarrow \mathbb{R}$ be such that $g^{-1}(0)$ is closed and (10) holds for every nonempty closed subset $K$ of $G$. Assume that $f$ belongs to $\mathcal{F}\left(\mathbb{B}_{\varepsilon}\left(g^{-1}(0)\right) \cap G\right)$ ) for some $\varepsilon>0$. If $x: \mathbb{R}_{+} \rightarrow \mathbb{R}^{N}$ is a global solution of (11) with $x\left(\mathbb{R}_{+}\right) \subset G$ and $g \circ x$ is weakly meagre, then statements (a)-(c) of Theorem 4.5 hold.

Proof. Fix $\delta$ in $(0, \varepsilon)$. By Proposition 5.3, $x$ is uniformly continuous on

$$
x^{-1}\left(\mathbb{B}_{\delta}\left(g^{-1}(0)\right)\right) \text {. }
$$

An application of Theorem 4.5 completes the proof.

In the following we use Theorem 5.4 to obtain a version of a well-known result on $\omega$-limit sets of solutions of nonautonomous ordinary differential equations. For a nonempty open subset $D$ of $\mathbb{R}^{N}$ and a continuously differentiable function $V: \mathbb{R}_{+} \times D \rightarrow \mathbb{R}$, we define $V_{f}: \mathbb{R}_{+} \times D \rightarrow \mathbb{R}$ (the derivative of $V$ with respect to (11) in the sense that $(d / d t) V(t, x(t))=V_{f}(t, x(t))$ along a solution $x$ of (11)) by

$$
V_{f}(t, \xi)=\frac{\partial V}{\partial t}(t, \xi)+\sum_{i=1}^{N} \frac{\partial V}{\partial \xi_{i}}(t, \xi) f_{i}(t, \xi)
$$

for all $(t, \xi)$ in $\mathbb{R}_{+} \times D$, where $f_{1}, \ldots, f_{N}$ denote the components of $f$. 
Corollary 5.6. Let $D$ be a nonempty open subset of $\mathbb{R}^{N}$, and let $V: \mathbb{R}_{+} \times D \rightarrow \mathbb{R}$ be continuously differentiable. Assume that $V$ satisfies the following two conditions:

(a) for each $\xi$ in $\operatorname{cl}(D)$ there exists a neighbourhood $U$ of $\xi$ such that $V$ is bounded from below on the set $\mathbb{R}_{+} \times(U \cap D)$;

(b) there exists a lower semicontinuous continuous function $W: \operatorname{cl}(D) \rightarrow \mathbb{R}_{+}$such that $V_{f}(t, \xi) \leq-W(\xi)$ for all $(t, \xi)$ in $\mathbb{R}_{+} \times D$.

Furthermore, assume that for every $\xi$ in $\mathrm{cl}(D)$ there exists a neighbourhood $U^{\prime}$ of $\xi$ such that $f$ belongs to $\mathcal{F}\left(U^{\prime} \cap D\right)$. Under these assumptions, if $x: \mathbb{R}_{+} \rightarrow \mathbb{R}^{N}$ is a global solution of (11) with $x\left(\mathbb{R}_{+}\right) \subset D$, then $\Omega(x) \subset W^{-1}(0)$.

Proof. If $\Omega(x)=\emptyset$ there is nothing to prove, so we assume that $\Omega(x) \neq \emptyset$. Since $(d / d t) V(t, x(t))=V_{f}(t, x(t))$ for all $t$ in $\mathbb{R}_{+}$, it follows from assumption (b) that the function $t \mapsto V(t, x(t))$ is nonincreasing, showing that the limit $l$ of $V(t, x(t))$ as $t \rightarrow \infty$ exists, where possibly $l=-\infty$. Let $\xi \in \Omega(x) \subset \operatorname{cl}(D)$. Then there exists a nondecreasing unbounded sequence $\left(t_{n}\right)$ in $\mathbb{R}_{+}$such that $\lim _{n \rightarrow \infty} x\left(t_{n}\right)=\xi$. By assumption (a) there exists a neighbourhood $U$ of $\xi$ such that $V$ is bounded from below on $\mathbb{R}_{+} \times(U \cap D)$. Now $x\left(\mathbb{R}_{+}\right) \subset D$, so there exists $n_{0}$ such that $x\left(t_{n}\right) \in U \cap D$ whenever $n \geq n_{0}$. Consequently, the nonincreasing sequence $\left(V\left(t_{n}, x\left(t_{n}\right)\right)\right)$ is bounded from below, showing that $l>-\infty$. Therefore

$$
\begin{aligned}
0 & \leq \int_{0}^{\infty}(W \circ x)(t) d t \leq-\int_{0}^{\infty} V_{f}(t, x(t)) d t \\
& =-\int_{0}^{\infty}(d / d t) V(t, x(t)) d t=V\left(0, x^{0}\right)-l<\infty,
\end{aligned}
$$

verifying that $W \circ x$ is in $L^{1}$, hence is weakly meagre. By assumption, for each $\xi$ in $\operatorname{cl}(D)$ there exists an open neighbourhood $U^{\prime}$ of $\xi$ such that $f$ belongs to $\mathcal{F}\left(U^{\prime} \cap D\right)$, implying that $f$ is a member of $\mathcal{F}\left(U^{\prime} \cap \mathrm{cl}(D)\right)$. Therefore, an application of Theorem 5.4 with $G=\operatorname{cl}(D)$ and $g=W$ establishes the claim.

Corollary 5.6 is essentially due to LaSalle [19] (see also [16, Satz 6.2, p. 140]). However, we point out that the assumption imposed on $f$ in Corollary 5.6 is weaker then that in [16] and [19], wherein it is required that, for every $\xi \operatorname{in~} \operatorname{cl}(D)$, there exists a neighbourhood $U^{\prime}$ of $\xi$ such that $f$ is bounded on the set $\mathbb{R}_{+} \times\left(U^{\prime} \cap D\right)$. Furthermore, we impose only lower semicontinuity on the function $W$ (in contrast to [16] and [19], wherein continuity of $W$ is assumed).

The next result is a consequence of Theorem 5.5. It shows, in particular, that under a mild assumption on $f$ every global $L^{p}$-solution of (11) converges to zero.

Corollary 5.7. Assume that there exists $\varepsilon>0$ such that $f$ belongs to $\mathcal{F}\left(\mathbb{B}_{\varepsilon}(0)\right)$, and let $x: \mathbb{R}_{+} \rightarrow \mathbb{R}^{N}$ be a global solution of (11). Then the following statements hold:

(a) If $\|x(\cdot)\|$ is weakly meagre, then $\lim _{t \rightarrow \infty} x(t)=0$.

(b) If $x$ belongs to $L^{p}$ for some $p$ in $(0, \infty)$, then $\lim _{t \rightarrow \infty} x(t)=0$.

Proof. If $\|x(\cdot)\|$ is weakly meagre, then an application of Theorem 5.5 with $G=\mathbb{R}^{N}$ and $g=\|\cdot\|$ shows that $\lim _{t \rightarrow \infty} x(t)=0$. This establishes statement (a). To prove statement (b), let $x$ be a member of $L^{p}$ for some $p$ in $(0, \infty)$. Then, by Proposition $4.2(\mathrm{a})$, the function $\|x(\cdot)\|$ is meagre and hence is weakly meagre. By part (a) of the present result, $\lim _{t \rightarrow \infty} x(t)=0$. 
Obviously, if (11) is autonomous (i.e., the differential equation in (11) has the form $\dot{x}(t)=f(x(t)))$, then the assumption that $f$ belongs to $\mathcal{F}\left(\mathbb{B}_{\varepsilon}(0)\right)$ for some $\varepsilon>0$ is trivially satisfied. Thus we may conclude that every weakly meagre global solution $t \mapsto x(t)$ of an autonomous ordinary differential equation converges to 0 as $t \rightarrow \infty$.

Example 5.8. In this example we describe a typical application of Theorem 5.5. In part (a) of the example we analyze a general class of second-order systems with nonautonomous "damping"; in part (b) we discuss a special case. We will return to this example in section 6 to refine the result further.

(a) Consider the second-order system

$$
\ddot{y}(t)+d(t, \dot{y}(t))+k(y(t))=0, \quad(y(0), \dot{y}(0))=\left(p^{0}, v^{0}\right) \in \mathbb{R}^{2},
$$

where $k: \mathbb{R} \rightarrow \mathbb{R}$ is a continuous function with the property that

$$
\lim _{|p| \rightarrow \infty} \int_{0}^{p} k(s) d s=\infty .
$$

We assume that $d: \mathbb{R}_{+} \times \mathbb{R} \rightarrow \mathbb{R}$ is a Carathéodory function satisfying the following conditions:

(i) $d(t, v) v \geq 0$ for all $(t, v)$ in $\mathbb{R}_{+} \times \mathbb{R}$;

(ii) for each bounded subset $K$ of $\mathbb{R}$ there exists a constant $b \geq 0$ such that $|d(t, v)| \leq b$ for all $(t, v)$ in $\mathbb{R}_{+} \times K$

(iii) the function $d_{*}: \mathbb{R} \rightarrow \mathbb{R}_{+}$given by $v \mapsto \inf _{t \in \mathbb{R}_{+}}|d(t, v)|$ is a Borel function such that $\inf _{v \in K} d_{*}(v)>0$ for every compact subset $C$ of $\mathbb{R}$ satisfying $C \cap \mathrm{cl}\left(d_{*}^{-1}(0)\right)=\emptyset$.

Since $d$ is a Carathéodory function, we know that the function $v \mapsto d(t, v)$ is continuous for each fixed $t$ in $\mathbb{R}_{+}$. Therefore it follows from assumption (i) that $d(t, 0)=0$ for all $t$ in $\mathbb{R}_{+}$, showing that 0 lies in $d_{*}^{-1}(0)$.

By standard existence theory, (12) has a solution and every solution has a maximal extension. As earlier, a solution $y$ of (12) is said to be global if $y$ is defined on $\mathbb{R}_{+}$.

Claim. For each $\left(p^{0}, v^{0}\right)$ in $\mathbb{R}^{2}$ every maximal solution $y$ of (12) is global and bounded, with bounded derivative $\dot{y}$. Moreover, $\dot{y}$ approaches $d_{*}^{-1}(0)$ and, if 0 is an isolated point of $d_{*}^{-1}(0)$, then $\lim _{t \rightarrow \infty} \dot{y}(t)=0$.

We proceed to establish this claim. Setting $x(t)=(y(t), \dot{y}(t))$, the second-order system (12) can be expressed in the equivalent form (11), where $f: \mathbb{R}_{+} \times \mathbb{R}^{2} \rightarrow \mathbb{R}^{2}$ and $x^{0}$ in $\mathbb{R}^{2}$ are given by

$$
f(t,(p, v))=(v,-k(p)-d(t, v)), \quad x^{0}=\left(p^{0}, v^{0}\right) .
$$

Define $V: \mathbb{R}^{2} \rightarrow \mathbb{R}$ by $(p, v) \mapsto \int_{0}^{p} k(s) d s+v^{2} / 2$. Observe that, by (13), $V$ is bounded from below and is such that, for every sequence $\left(\xi_{n}\right)$ in $\mathbb{R}^{2}$,

$$
\left\|\xi_{n}\right\| \rightarrow \infty \quad \text { as } n \rightarrow \infty \quad \Longrightarrow \quad V\left(\xi_{n}\right) \rightarrow \infty \quad \text { as } n \rightarrow \infty
$$

Let $x=(y, \dot{y}):\left[0, \omega_{x}\right) \rightarrow \mathbb{R}^{2}$ be a maximal solution of (11) (with $f$ and $x^{0}$ given by (14)). Then for almost all $t$ in $\left[0, \omega_{x}\right)$,

$$
\frac{d}{d t} V(x(t)) \leq-d(t, \dot{y}(t)) \dot{y}(t)=-|d(t, \dot{y}(t))||\dot{y}(t)| \leq-d_{*}(\dot{y}(t))|\dot{y}(t)| \leq 0,
$$


wherein we have invoked assumption (i) on $d$. Consequently, $c \leq V(x(t)) \leq V\left(x^{0}\right)$ for all $t$ in $\left[0, \omega_{x}\right)$ and for some constant $c$. Combining this with (15), we infer that $x$ is bounded, so $\omega_{x}=\infty$. Moreover, $l=\lim _{t \rightarrow \infty} V(x(t))$ exists in $\mathbb{R}$ and hence

$$
0 \leq \int_{0}^{\infty} d_{*}(\dot{y}(t))|\dot{y}(t)| d t \leq V\left(x^{0}\right)-l<\infty .
$$

Since $x$ is bounded, there exists a compact interval $I$ such that $x\left(\mathbb{R}_{+}\right) \subset I \times I$. Define $g: I \times I \rightarrow \mathbb{R}_{+}$as follows

$$
g(p, v)= \begin{cases}0 & \text { if }(p, v) \in I \times\left(\operatorname{cl}\left(d_{*}^{-1}(0)\right) \cap I\right) \\ d_{*}(v)|v| & \text { otherwise }\end{cases}
$$

Then $g^{-1}(0)=I \times\left(\operatorname{cl}\left(d_{*}^{-1}(0)\right) \cap I\right)$. In particular, $g^{-1}(0)$ is closed. It follows from assumption (iii) on $d$ that $g$ satisfies (10) for every nonempty closed subset $K$ of $I \times I$. Moreover, assumption (ii) on $d$ implies that $f$ belongs to $\mathcal{F}(B)$ for every bounded subset $B$ of $\mathbb{R}^{2}$, so $f$ is in $\mathcal{F}\left(\mathbb{B}_{\varepsilon}\left(g^{-1}(0)\right) \cap(I \times I)\right)$ for $\varepsilon>0$. By (16), $g \circ x$ is weakly meagre, and thus we may appeal to Theorem 5.5(b) to conclude that $x=(y, \dot{y})$ approaches $g^{-1}(0)$. Consequently, $\dot{y}$ approaches $d_{*}^{-1}(0)$. Finally, assume that 0 is an isolated point of $d_{*}^{-1}(0)$ and hence of $\operatorname{cl}\left(d_{*}^{-1}(0)\right)$. Now $\dot{y}$ is continuous and bounded. By Lemma 2.1(c), the $\omega$-limit set $\Omega(\dot{y})$ of $\dot{y}$ is nonempty, compact, and connected, is approached by $\dot{y}$, and is the smallest closed set approached by $\dot{y}$. Consequently, there exists $\varepsilon>0$ such that either $\Omega(\dot{y}) \subset(-\infty,-\varepsilon]$ or $\Omega(\dot{y}) \subset[\varepsilon, \infty)$ or $\Omega(\dot{y})=\{0\}$. Since $y$ is bounded, it follows that the first two alternatives are impossible. Therefore, we conclude that $\lim _{t \rightarrow \infty} \dot{y}(t)=0$, completing the proof of the claim.

(b) For purposes of illustration and to provide a connection with the material in section 6 , we choose a specific example wherein $d$ is such that the associated function $d_{*}$ is discontinuous. In particular, consider $d: \mathbb{R}_{+} \times \mathbb{R} \rightarrow \mathbb{R}$ given by

$d(t, v)= \begin{cases}v & \text { if }(t, v) \in \mathbb{R}_{+} \times[-1,1], \\ \operatorname{sgn}(v) \max \{0,1-(t+1)(|v|-1)\} & \text { if }(t, v) \in \mathbb{R}_{+} \times\{v \in \mathbb{R}:|v|>1\}\end{cases}$

where $\operatorname{sgn}(v)$ denotes the sign of $v$. For generic $t$ in $\mathbb{R}_{+}$, the graph of $v \mapsto d(t, v)$ is depicted in Figure 3. Observe that $d$ satisfies assumptions (i) and (ii). Furthermore, it

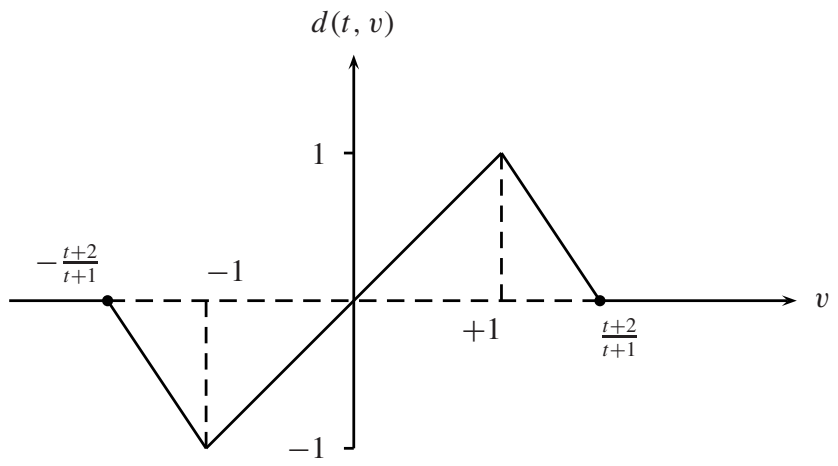

Figure 3. 
is readily verified that the function $d_{*}: v \mapsto \inf _{t \in \mathbb{R}_{+}}|d(t, v)|$ can be expressed as

$$
d_{*}(v)= \begin{cases}|v| & \text { if } v \in[-1,1] \\ 0 & \text { if }|v|>1\end{cases}
$$

Clearly, $d_{*}$ is piecewise continuous (with jump discontinuities at $v= \pm 1$ ) and therefore is a Borel function. Moreover, $\operatorname{cl}\left(d_{*}^{-1}(0)\right)=\{v \in \mathbb{R}:|v| \geq 1\} \cup\{0\}$ and it is clear that $\inf _{v \in C} d_{*}(v)>0$ for every compact subset $C$ of $\mathbb{R}$ with $C \cap \operatorname{cl}\left(d_{*}^{-1}(0)\right)=\emptyset$, showing that assumption (iii) is satisfied. Since, trivially, 0 is an isolated point of $d_{*}^{-1}(0)$, it follows from part (a) that $\lim _{t \rightarrow \infty} \dot{y}(t)=0$. In section 6 (see Example 6.3) we will further refine this result to conclude that $(y, \dot{y})$ approaches the set $k^{-1}(0) \times\{0\}$.

6. APPLICATIONS TO AUTONOMOUS DIFFFERENTIAL INCLUSIONS. In section 5, we investigated the behaviour of systems within the framework of ordinary differential equations with Carathéodory right-hand sides. However, there are many meaningful situations wherein this framework is inadequate for purposes of analysis of dynamic behaviour. A prototypical example is that of a mechanical system with Coulomb friction, which, formally, yields a differential equation with discontinuous right-hand side (one such system is analyzed in Example 6.9). Other examples permeate control theory and applications: a canonical case is a discontinuous feedback strategy associated with an on-off or switching device. Such discontinuous phenomena can be handled mathematically by embedding the discontinuities in set-valued maps, giving rise to the study of differential inclusions of the form $\dot{x} \in F(x)$, on which there is a growing literature (see, for example, [2], [6], [7], [10], [12], [29]). The next goal is to extend our investigations on ordinary differential equations to differential inclusions. We first assemble some basic definitions and results.

Let $\mathcal{U}$ denote the class of set-valued maps $\xi \mapsto F(\xi) \subset \mathbb{R}^{N}$, defined on $\mathbb{R}^{N}$, that are upper semicontinuous at each $\xi$ in $\mathbb{R}^{N}$ and take nonempty convex compact values. We recall that a set-valued map $F$ is upper semicontinuous at $\xi$ in $\mathbb{R}^{N}$ if for each $\varepsilon>0$ there exists $\delta>0$ such that $F\left(\xi^{\prime}\right) \subset \mathbb{B}_{\varepsilon}\left(F(\xi)\right.$ ) for all $\xi^{\prime}$ in $\mathbb{B}_{\delta}(\xi)$ (see Figure 4).
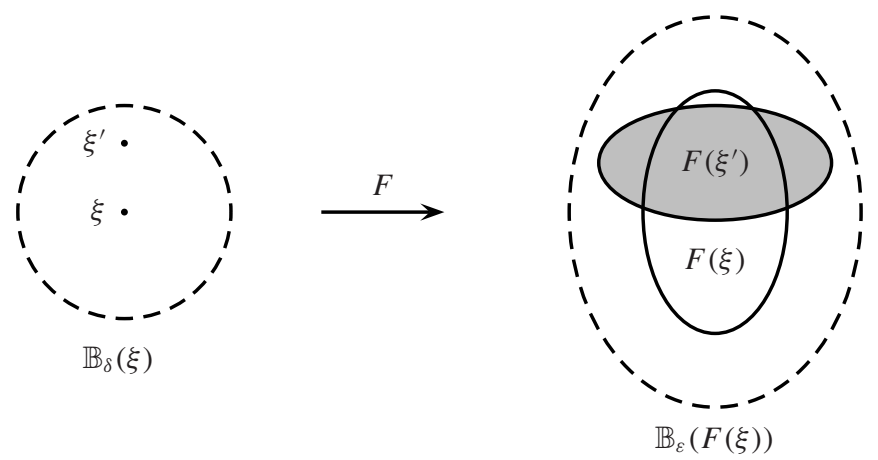

Figure 4 .

We consider next the initial-value problem for an autonomous differential inclusion corresponding to a mapping $F$ in $\mathcal{U}$ :

$$
\dot{x}(t) \in F(x(t)), \quad x(0)=x^{0} \in \mathbb{R}^{N} .
$$

We will study asymptotic properties of solutions of (18), where by a solution on $[0, \omega)$ we mean a locally absolutely continuous function $x:[0, \omega) \rightarrow \mathbb{R}^{N}$ satisfying (18) 
almost everywhere on $[0, \omega)$. A solution on $\mathbb{R}_{+}$is again called a global solution. The concept of a maximal solution of (18) is the natural analogue of that for differential equations (see section 5). We record some well-known facts in Lemmas 6.1 and 6.2, which represent a distillation of results in, for example, [2], [12], and [26].

Lemma 6.1. Let $F$ belong to $\mathcal{U}$, and let $x^{0}$ be a point in $\mathbb{R}^{N}$. Then the initial value problem (18) has a solution, and every solution $x$ can be extended to a maximal solution with maximal interval of existence $\left[0, \omega_{x}\right)$. If $\omega_{x}<\infty$, then $x$ is unbounded.

It follows from Lemma 6.1 that bounded maximal solutions of (18) are global. With respect to (18), a nonempty subset $A$ of $\mathbb{R}^{N}$ is said to be weakly invariant if, for each $x^{0}$ in $A$, (18) has at least one maximal solution $x:\left[0, \omega_{x}\right) \rightarrow \mathbb{R}^{N}$ with $x(t)$ in $A$ for all $t$ in $\left[0, \omega_{x}\right)$.

Lemma 6.2. Let $F$ belong to $\mathcal{U}$. If $x: \mathbb{R}_{+} \rightarrow \mathbb{R}^{N}$ is a bounded global solution of (18), then $\Omega(x)$ is nonempty, compact, and connected, is approached by $x$ (and is the smallest closed set so approached), and is weakly invariant with respect to (18).

Example 6.3. Let us revisit the special case (b) of Example 5.8. Let $f$ and $x^{0}$ be given by (14), and let $x=(y, \dot{y}):\left[0, \omega_{x}\right) \rightarrow \mathbb{R}^{N}$ be a maximal solution of (11). We already know that $\omega_{x}=\infty$, that $x$ is bounded, and that $\Omega(x)$ is a nonempty subset of $\mathbb{R} \times\{0\}$. Defining a set-valued map $\Delta$ on $\mathbb{R}$ by

$$
\Delta(v)= \begin{cases}\{v\} & \text { if }|v| \leq 1 \\ {[-1,1]} & \text { if }|v|>1\end{cases}
$$

we observe that $d(t, v)$ lies in $\Delta(v)$ for all $(t, v)$ in $\mathbb{R}_{+} \times \mathbb{R}$, where $d$ is defined by (17). On $\mathbb{R} \times \mathbb{R}$, define the set-valued map $F$ by

$$
F(p, v)=\{v\} \times\{-k(p)-w: w \in \Delta(v)\} .
$$

Evidently, $F$ is a member of $\mathcal{U}$ and $f(t,(p, v))$ lies in $F(p, v)$ for all $(t,(p, v))$ in $\mathbb{R}_{+} \times \mathbb{R}^{2}$. Therefore, the solution $x$ of $(11)$ is a fortiori a solution of the differential inclusion $\dot{x}(t) \in F(x(t))$. By Lemma 6.2, $\Omega(x)$ is weakly invariant with respect to that differential inclusion. Since $x$ approaches $\Omega(x)$, a subset of $\mathbb{R} \times\{0\}$, it follows that $x$ must approach the largest subset $E$ of $\mathbb{R} \times\{0\}$ that is weakly invariant with respect to the differential inclusion. Consider a point $(p, v)$ in $E$. By the weak invariance of $E$, there exists a maximal solution $(z, \dot{z})$ of the differential inclusion such that $(z(0), \dot{z}(0))=(p, v)=(p, 0)$ and $(z(t), \dot{z}(t))$ belongs to $E$ for all $t$ in $\left[0, \omega_{(z, \dot{z})}\right)$. Therefore, $z(t)=p$ for all $t$ in $\mathbb{R}_{+}$and, noting that $\Delta(0)=\{0\}$, we have

$$
(0,0)=(\dot{z}(t), \ddot{z}(t)) \in F(z(t), \dot{z}(t))=F(p, 0)=\{(0,-k(p))\}
$$

for almost all $t$ in $\left[0, \omega_{(z, \dot{z})}\right)$, whence $k(p)=0$. Thus, $E \subset k^{-1}(0) \times\{0\}$ and so $x$ approaches the set $k^{-1}(0) \times\{0\}$. Finally, note that, if $k^{-1}(0)$ is totally disconnected, then $x$ approaches an equilibrium of the nonautonomous differential equation (11) (with $f$ and $x^{0}$ given by (14)).

The following proposition shows that, under suitable local boundedness assumptions on $F$, the solutions of (18) satisfy the uniform continuity assumptions required for an application of Theorems 4.4 and 4.5. For a subset $A$ of $\mathbb{R}^{N}$ and for a member $F$ of $\mathcal{U}$ we denote (in a slight abuse of notation) the set $\cup_{a \in A} F(a)$ by $F(A)$. 
Proposition 6.4. Let $A$ and $B$ be subsets of $\mathbb{R}^{N}$, and let $F$ belong to $\mathcal{U}$. Assume that $F\left(\mathbb{B}_{\varepsilon}(A) \cap B\right)$ is bounded for some $\varepsilon>0$ and that $x: \mathbb{R}_{+} \rightarrow \mathbb{R}^{N}$ is a global solution of $(18)$ with $x\left(\mathbb{R}_{+}\right) \subset B$. Then $x$ is uniformly continuous on $x^{-1}(A)$.

Proof. If $x^{-1}(A)=\emptyset$, then the assertion holds trivially. Assume that $x^{-1}(A) \neq \varnothing$, and let $\delta$ in $(0, \varepsilon)$ be arbitrary. Define $\theta=\sup \left\{\|v\|: v \in F\left(\mathbb{B}_{\varepsilon}(A) \cap B\right)\right\}$, and let $\tau>0$ be sufficiently small that $\tau \theta \leq \delta$. Adopting an argument similar to that used in the proof of Proposition 5.3, it can be shown that $\left\|x\left(t_{2}\right)-x\left(t_{1}\right)\right\| \leq \delta$ for all $t_{1}$ and $t_{2}$ in $x^{-1}(A)$ with $0 \leq t_{2}-t_{1} \leq \tau$, proving that $x$ is uniformly continuous on $x^{-1}(A)$.

We now use Theorems 4.4 and 4.5 to derive counterparts of Theorems 5.4 and 5.5 for differential inclusions.

Theorem 6.5. Let $G$ be a nonempty closed subset of $\mathbb{R}^{N}$, let $g: G \rightarrow \mathbb{R}$ have the property that each $\xi$ in $G$ for which $g(\xi) \neq 0$ has a neighbourhood $U$ such that (7) holds, and let $F$ belong to $\mathcal{U}$. If $x: \mathbb{R}_{+} \rightarrow \mathbb{R}^{N}$ is a global solution of (18) with $x\left(\mathbb{R}_{+}\right) \subset G$ and $g \circ x$ is weakly meagre, then statements (a) and (d) of Theorem 4.4 hold. Moreover, the following statements are true:

( $\left.\mathrm{b}^{\prime}\right)$ If $g^{-1}(0)$ is bounded and $\Omega(x) \neq \emptyset$, then $x$ is bounded and $x$ approaches the largest subset of $g^{-1}(0)$ that is weakly invariant with respect to (18).

$\left(\mathrm{c}^{\prime}\right)$ If $x$ is bounded, then $g^{-1}(0) \neq \emptyset$ and $x$ approaches the largest subset of $g^{-1}(0)$ that is weakly invariant with respect to (18).

Proof. Let $\xi$ in $G$ be such that $g(\xi) \neq 0$. By hypothesis, there exists $\varepsilon>0$ such that (7) holds with $U=\mathbb{B}_{\varepsilon}(\xi)$. By the upper semicontinuity of $F$, together with the compactness of its values, $F\left(\mathbb{B}_{\varepsilon}(U) \cap G\right)$ is bounded (see [2, Proposition 3, p. 42]). By Proposition 6.4, $x$ is uniformly continuous on $x^{-1}(U)$. Therefore, the hypotheses of Theorem 4.4 are satisfied, so statements (a)-(d) thereof hold. Combining statements (b) and (c) of Theorem 4.4 with the weak invariance of $\Omega(x)$ yields statements $\left(b^{\prime}\right)$ and $\left(\mathrm{c}^{\prime}\right)$.

Theorem 6.6. Let $G$ be a nonempty closed subset of $\mathbb{R}^{N}$, let $g: G \rightarrow \mathbb{R}$ be such that $g^{-1}(0)$ is closed and (10) holds for every nonempty closed subset $K$ of $G$, and let $F$ belong to $\mathcal{U}$. Assume that $F\left(\mathbb{B}_{\varepsilon}\left(g^{-1}(0)\right) \cap G\right)$ is bounded for some $\varepsilon>0$. If $x: \mathbb{R}_{+} \rightarrow \mathbb{R}^{N}$ is a global solution of $(18)$ with $x\left(\mathbb{R}_{+}\right) \subset G$ and $g \circ x$ is weakly meagre, then statements (a) and (c) of Theorem 4.5 hold. Moreover, the following also holds:

$\left(\mathrm{b}^{\prime}\right)$ If $g^{-1}(0)$ is bounded, then $x$ is bounded and $x$ approaches the largest subset of $g^{-1}(0)$ that is weakly invariant with respect to (18).

Proof. Fix $\delta$ in $(0, \varepsilon)$. By Proposition $6.4, x$ is uniformly continuous on

$$
x^{-1}\left(\mathbb{B}_{\delta}\left(g^{-1}(0)\right)\right) \text {. }
$$

It follows immediately from Theorem 4.5 that statements (a)-(c) thereof hold. Assuming that $g^{-1}(0)$ is bounded, a combination of statements (b) of Theorem 4.5 with the weak invariance of $\Omega(x)$ yields statement $\left(b^{\prime}\right)$.

If there exists a locally Lipschitz function $f: \mathbb{R}^{N} \rightarrow \mathbb{R}^{N}$ such that $F(x)=\{f(x)\}$ (that is, the differential inclusion (18) "collapses" to an autonomous differential equation that for every $x^{0}$ in $\mathbb{R}^{N}$ has a unique solution satisfying $x(0)=x^{0}$ ), then the 
conclusions of Theorems 6.5 and 6.6 remain true when every occurence of "weakly invariant" is replaced with "invariant". We mention that precursors of Theorems 6.5 and 6.6 have appeared in [11] and [27].

We now use Theorem 6.6 to generalize LaSalle's invariance principle (see Corollary 3.4) to differential inclusions.

Corollary 6.7. Let $D$ be a nonempty open subset of $\mathbb{R}^{N}$, let $V: D \rightarrow \mathbb{R}$ be continuously differentiable, let $F$ belong to $\mathcal{U}$, and set $V_{F}(\xi)=\max _{y \in F(\xi)}\langle\nabla V(\xi), y\rangle$ for all $\xi$ in D. Let $x: \mathbb{R}_{+} \rightarrow \mathbb{R}^{N}$ be a (global) solution of (18) and assume that there exists a compact subset $G$ of $\mathbb{R}^{N}$ such that $x\left(\mathbb{R}_{+}\right) \subset G \subset D$. If $V_{F}(\xi) \leq 0$ for all $\xi$ in $G$, then $x$ approaches the largest subset of $V_{F}^{-1}(0) \cap G$ that is weakly invariant with respect to $(18)$.

Proof. For later convenience, we first show that the function $V_{F}: D \rightarrow \mathbb{R}$ is upper semicontinuous. Let $\left(\xi_{n}\right)$ be a convergent sequence in $D$ with limit $\xi$ in $D$. Define $l=\limsup _{n \rightarrow \infty} V_{F}\left(\xi_{n}\right)$. From $\left(V_{F}\left(\xi_{n}\right)\right)$ extract a subsequence $\left(V_{F}\left(\xi_{n_{k}}\right)\right)$ with $V_{F}\left(\xi_{n_{k}}\right) \rightarrow l$ as $k \rightarrow \infty$. For each $k$, let $y_{k}$ be a maximizer of the continuous function $y \mapsto\left\langle\nabla V\left(\xi_{n_{k}}\right), y\right\rangle$ over the compact set $F\left(\xi_{n_{k}}\right)$, so $V_{F}\left(\xi_{n_{k}}\right)=\left\langle\nabla V\left(\xi_{n_{k}}\right), y_{k}\right\rangle$. Let $\varepsilon>0$ be arbitrary. By the upper semicontinuity of $F, F\left(\xi_{n_{k}}\right) \subset \mathbb{B}_{\varepsilon}(F(\xi))$ for all sufficiently large $k$. Since $y_{k}$ lies in $F\left(\xi_{n_{k}}\right), F(\xi)$ is compact, and $\varepsilon>0$ is arbitrary, we infer that $\left(y_{k}\right)$ has a subsequence (which we do not relabel) converging to a point $y^{*}$ in $F(\xi)$. Therefore,

$$
\limsup _{n \rightarrow \infty} V_{F}\left(\xi_{n}\right)=l=\lim _{k \rightarrow \infty} V_{F}\left(\xi_{n_{k}}\right)=\lim _{k \rightarrow \infty}\left\langle\nabla V\left(\xi_{n_{k}}\right), y_{k}\right\rangle=\left\langle\nabla V(\xi), y^{*}\right\rangle \leq V_{F}(\xi),
$$

confirming that $V_{F}$ is upper semicontinuous.

Evidently,

$$
\frac{d}{d t} V(x(t))=\langle\nabla V(x(t)), \dot{x}(t)\rangle \leq V_{F}(x(t)) \leq 0
$$

for almost every $t$ in $\mathbb{R}_{+}$, which leads to

$$
V(x(t))-V(x(0)) \leq \int_{0}^{t} V_{F}(x(s)) d s \leq 0
$$

for all $t$ in $\mathbb{R}_{+}$. Since $x$ is bounded, we conclude that the function $t \mapsto \int_{0}^{t} V_{F}(x(s)) d s$ is bounded from below. But this function is also nonincreasing (because $V_{F} \leq 0$ on $G$ ), which ensures that $\lim _{t \rightarrow \infty} \int_{0}^{t} V_{F}(x(s)) d s$ exists and is finite. Consequently, $V_{F} \circ x$ is an $L^{1}$-function, showing that $V_{F} \circ x$ is weakly meagre. Since $V_{F}$ is upper semicontinuous and $V_{F} \leq 0$ on $G$, the function $G \rightarrow \mathbb{R}$ given by $\xi \mapsto\left|V_{F}(\xi)\right|$ is lower semicontinuous. Therefore, each $\xi$ in $G$ with $V_{F}(\xi) \neq 0$ has a neighbourhood $U$ such that $\inf \left\{\left|V_{F}(w)\right|: w \in G \cap U\right\}>0$. By statement $\left(\mathrm{c}^{\prime}\right)$ of Theorem 6.5 (with $g=\left.V_{F}\right|_{G}$ ) it follows that $x$ approaches the largest subset of $V_{F}^{-1}(0) \cap G$ that is weakly invariant with respect to (18).

In Corollary 6.7, it is assumed that the solution $x$ is global and has trajectory in some compact subset $G$ of $D$. These conditions may be removed at the expense of strengthening the conditions on $V$ by assuming that its sublevel sets are bounded and that $V_{F}(\xi) \leq 0$ for all $\xi$ in $D$. 
Corollary 6.8. Let $D, V, F$, and $V_{F}$ be as in Corollary 6.7. Assume that the sublevel sets of $V$ are bounded and that $V_{F}(\xi) \leq 0$ for all $\xi$ in $D$. If $x:\left[0, \omega_{x}\right) \rightarrow \mathbb{R}^{N}$ is a maximal solution of (18) such that $\operatorname{cl}\left(x\left(\left[0, \omega_{x}\right)\right)\right) \subset D$, then $x$ is bounded, $\omega_{x}=\infty$, and $x$ approaches the largest subset of $V_{F}^{-1}(0)$ that is weakly invariant with respect to $(18)$.

Proof. Since $(d / d t) V(x(t))=V_{F}(x(t)) \leq 0$ for almost all $t$ in $\left[0, \omega_{x}\right)$, we have the counterpart of (19): $V(x(t)) \leq V(x(0))$ for all $t$ in $\left[0, \omega_{x}\right)$. Since the sublevel sets of $V$ are bounded, it follows that $x$ is bounded. By Lemma $6.1, \omega_{x}=\infty$. An application of Corollary 6.7, with $G=\operatorname{cl}\left(x\left(\mathbb{R}_{+}\right)\right)$, completes the proof.

Example 6.9. In this example we describe a typical application of Corollary 6.8. In part (a) of the example we analyze a general class of second-order differential inclusions; in part (b) we discuss a special case, a mechanical system subject to friction of Coulomb type.

(a) Let $k: \mathbb{R} \rightarrow \mathbb{R}$ be as in Example 5.8, that is, $k$ is continuous with property (13). Let $(p, v) \mapsto C(p, v) \subset \mathbb{R}$ be upper semicontinuous with nonempty, convex, compact values and with the property that, for all $(p, v)$ in $\mathbb{R}^{2}$,

$$
C_{*}(p, v):=\max \{v w: w \in C(p, v)\} \leq 0 .
$$

Consider the system

$$
\ddot{y}(t)+k(y(t)) \in C(y(t), \dot{y}(t)), \quad(y(0), \dot{y}(0))=\left(p^{0}, v^{0}\right) \in \mathbb{R}^{2} .
$$

Setting $x(t)=(y(t), \dot{y}(t))$, the second-order initial-value problem (21) can be expressed in the equivalent form

$$
\dot{x}(t) \in F(x(t)), \quad x(0)=x^{0}=\left(p^{0}, v^{0}\right) \in \mathbb{R}^{2},
$$

where the set-valued map $F$ in $\mathcal{U}$ is given by

$$
F(p, v)=\{v\} \times\{-k(p)+w: w \in C(p, v)\} .
$$

By Lemma 6.1, (22) has a solution and every solution can be extended to a maximal solution; moreover, every bounded maximal solution is global.

Claim A. For each $x^{0}=\left(p^{0}, v^{0}\right)$ in $\mathbb{R}^{2}$, every maximal solution $x=(y, \dot{y})$ of (22) is bounded (hence, global) and approaches the largest subset $E$ of $C_{*}^{-1}(0)$ that is weakly invariant with respect to (22).

To establish this claim, we define (as in Example 5.8) $V: \mathbb{R}^{2} \rightarrow \mathbb{R}$ by

$$
V(p, v)=\int_{0}^{p} k(s) d s+v^{2} / 2 .
$$

Observe that by (13) $V$ is such that, for every sequence $\left(\xi_{n}\right)$ in $\mathbb{R}^{2},(15)$ holds and, as a result, every sublevel set of $V$ is bounded. Moreover,

$$
V_{F}(p, v)=\max _{\theta \in F(p, v)}\langle\nabla V(p, v), \theta\rangle=C_{*}(p, v) \leq 0 \quad\left((p, v) \in \mathbb{R}^{2}\right) .
$$

Let $x^{0}=\left(p^{0}, v^{0}\right)$ be a point in $\mathbb{R}^{2}$, and let $x=(y, \dot{y})$ be a maximal solution of (22). An application of Corollary 6.8, with $D=\mathbb{R}^{N}$, completes the proof of Claim A. 
(b) As a particular example, the mechanical system depicted in Figure 5, wherein a mass is subject to a friction force of Coulomb type on a rough surface of length $2 L$ (where $L>0$ ) and is friction free off the surface, may be represented by a secondorder autonomous differential inclusion of the form (21).

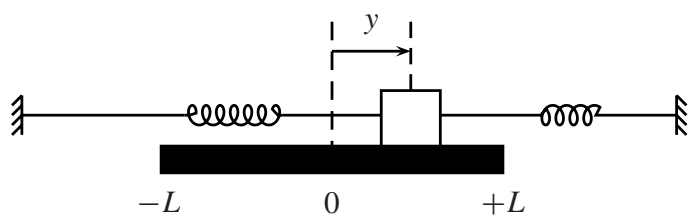

Figure 5.

In this specific example, the function $k$ (continuous with property (13)) corresponds to the spring force and is assumed to be such that $k^{-1}(0)=\{0\}$. The (upper semicontinuous) set-valued map $C$, which models the Coulomb friction effects, is given by

$$
C(p, v)= \begin{cases}\{-\operatorname{sgn}(v)\} & \text { if }|p|<L, v \neq 0 \\ {[-1,1]} & \text { if }|p| \leq L, v=0 \\ {[-1,0]} & \text { if }|p|=L, v>0 \\ {[0,1]} & \text { if }|p|=L, v<0 \\ \{0\} & \text { if }|p|>L, v \in \mathbb{R}\end{cases}
$$

Claim B. For each $x^{0}=\left(p^{0}, v^{0}\right)$ in $\mathbb{R}^{2}$, every maximal solution $x=(y, \dot{y})$ of $(22)$ (with $F$ and $C$ given by (23) and (24)) is bounded, is global, and approaches the set $\left([-L, L] \cap k^{-1}([-1,1])\right) \times\{0\}$.

To prove this claim, we first note that in this case the function $C_{*}$ (defined in (20)) is given by

$$
C_{*}(p, v)= \begin{cases}-|v| & \text { if }|p|<L, \\ 0 & \text { if }|p| \geq L .\end{cases}
$$

Therefore, $C_{*}^{-1}(0)=S_{1} \cup S_{2} \cup S_{3}$, where

$$
\begin{aligned}
& S_{1}=[-L, L] \times\{0\}, \\
& S_{2}=\left\{(p, v) \in \mathbb{R}^{2}:|p|=L, v \neq 0\right\}, \\
& S_{3}=\left\{(p, v) \in \mathbb{R}^{2}:|p|>L\right\} .
\end{aligned}
$$

By Claim A, for each $x^{0}=\left(p^{0}, v^{0}\right)$ in $\mathbb{R}^{2}$, every maximal solution $x=(y, \dot{y})$ of $(22)$ is bounded, is global, and approaches the largest subset $E$ of $S_{1} \cup S_{2} \cup S_{3}$ that is weakly invariant with respect to (22). To conclude Claim B, it suffices to show that

$$
E \subset\left([-L, L] \cap k^{-1}([-1,1])\right) \times\{0\} .
$$

To this end, we first show that

$$
E \cap\left(S_{2} \cup S_{3}\right)=\emptyset .
$$


By (21), the following observations are immediate:

(i) if $\left(p^{0}, v^{0}\right)$ is in $S_{3}$, then $(y(t), \dot{y}(t))$ is in $S_{3}$ for all sufficiently small $t>0$;

(ii) if $\left(p^{0}, v^{0}\right)$ is in $S_{2}$ and $p^{0} v^{0}>0$, then $(y(t), \dot{y}(t))$ is in $S_{3}$ for all sufficiently small $t>0$

(iii) if $\left(p^{0}, v^{0}\right)$ is in $S_{2}$ and $p^{0} v^{0}<0$, then $(y(t), \dot{y}(t)) \notin S_{1} \cup S_{2} \cup S_{3}$ holds for all sufficiently small $t>0$.

Seeking a contradiction, suppose that (25) does not hold. Then there exists a point $(p, v)$ in $E \cap\left(S_{2} \cup S_{3}\right)$ and a global bounded solution $(z, \dot{z})$ such that $(z(0), \dot{z}(0))=$ $(p, v)$ and $(z(t), \dot{z}(t)) \in E \subset S_{1} \cup S_{2} \cup S_{3}$ for all $t$ in $\mathbb{R}_{+}$. If $(p, v)$ belongs to $S_{2}$, then $p v>0$, for otherwise observation (iii) leads to the contradiction that $(z(t), \dot{z}(t)) \notin$ $S_{1} \cup S_{2} \cup S_{3}$ for all sufficiently small $t>0$. Therefore, by observations (i) and (ii), $(z(t), \dot{z}(t))$ lies in $S_{3}$ for all sufficiently small $t>0$. We consider the following two alternatives:

$$
\begin{array}{lll}
(\alpha) & (z(t), \dot{z}(t)) \in S_{3} & \text { for all } t>0 \\
(\beta) & \left(z\left(t_{0}\right), \dot{z}\left(t_{0}\right)\right) \notin S_{3} & \text { for some } t_{0}>0 .
\end{array}
$$

We show that both lead to contradictions.

First, suppose that alternative $(\alpha)$ holds. Then $|z(t)|>L>0$ for all $t>0$. Moreover, $z$ is bounded. Since $k$ and $z$ are continuous and $k^{-1}(0)=\{0\}$, we conclude that there exists $\varepsilon>0$ such that either $k(z(t)) \geq \varepsilon$ for all $t>0$ or $k(z(t)) \leq-\varepsilon$ for all $t>0$. On noting that $\ddot{z}(t)=-k(z(t))$ for all $t>0$, we obtain a contradiction to the boundedness of $\dot{z}$.

Second, suppose that alternative $(\beta)$ applies. Define $\tau=\inf \{t>0:(z(t), \dot{z}(t)) \notin$ $\left.S_{3}\right\}>0$. Then $|z(\tau)|=L$ and

$$
z(\tau) p>0
$$

Since $\ddot{z}(t)=-k(z(t))$ for all $t$ in $(0, \tau)$, a straightforward calculation shows that $V(z(t), \dot{z}(t))$ is constant on the interval $[0, \tau]$ and thus that

$$
\int_{0}^{z(t)} k(s) d s+(\dot{z}(t))^{2} / 2=\int_{0}^{p} k(s) d s+v^{2} / 2 \quad(t \in[0, \tau]) .
$$

If $(p, v)$ is in $S_{2}$, then $z(\tau)=p$ and $v \neq 0$, so by (27) $\dot{z}(\tau) \neq 0$. If $(p, v)$ is in $S_{3}$, then $|p|>|z(\tau)|$. Combining this with (26) and the inequality $k(\xi) \xi>0$ for all nonzero real $\xi$ (the latter being a consequence of (13), the continuity of $k$, and the fact that $\left.k^{-1}(0)=\{0\}\right)$, it follows again from $(27)$ that $\dot{z}(\tau) \neq 0$. Therefore, $(z(\tau), \dot{z}(\tau))$ belongs to $S_{2}$. Furthermore, it is clear that $z(\tau) \dot{z}(\tau)<0$, and therefore, by observation (iii), there exists $\delta>0$ such that $(z(t), \dot{z}(t)) \notin S_{1} \cup S_{2} \cup S_{3}$ holds for all $t$ in $(\tau, \tau+\delta)$. This contradicts the fact that $(z(t), \dot{z}(t)) \in E \subset S_{1} \cup S_{2} \cup S_{3}$ for all $t$ in $\mathbb{R}_{+}$. We can now conclude that (25) holds.

By (25), we have $E \subset S_{1}$. If $(p, v)$ is a point of $E$, then $|p| \leq L$ and $v=0$. By the weak invariance of $E$, there exists a maximal solution $(z, \dot{z})$ of $(22)$ satisfying $(z(0), \dot{z}(0))=(p, 0)$ that never leaves $E$. Consequently, $(z(t), \dot{z}(t)) \equiv(p, 0)$. By (21), $k(p)$ belongs to $C(p, 0)=[-1,1]$, whence $p$ is in $k^{-1}([-1,1])$. As a result, $E \subset\left([-L, L] \cap k^{-1}([-1,1])\right) \times\{0\}$. Now $x$ approaches $E$, so a fortiori $x$ approaches the set $\left([-L, L] \cap k^{-1}([-1,1])\right) \times\{0\}$. 


\section{APPENDIX.}

Example 7.1. We construct a continuous nonmeagre function that is both weakly meagre and Riemann integrable.

Consider the continuous function $y: \mathbb{R}_{+} \rightarrow \mathbb{R}$ given by $t \mapsto \sum_{n \in \mathbb{N}} y_{n}(t)$, where for each $n$ in $\mathbb{N}, y_{n}: \mathbb{R}_{+} \rightarrow[-1,1]$ is the piecewise linear continuous function, compactly supported on $[n, n+1 / n]$, whose graph is shown in Figure 6 (wherein the corners occur at $t=n+k /(5 n)$ for $k=0, \ldots, 5)$.

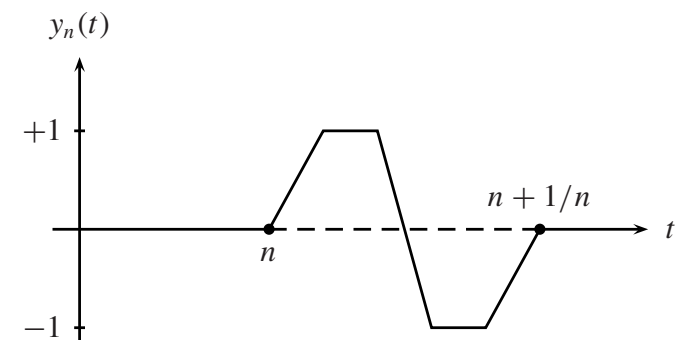

Figure 6.

To demonstrate the Riemann integrability of $y$, note that for all $t$ in $[1, \infty)$ we have

$$
\left|\int_{0}^{t} y(s) d s\right| \leq \int_{\lfloor t\rfloor}^{t} y_{\lfloor t\rfloor}(s) d s<\frac{1}{2\lfloor t\rfloor},
$$

where $\lfloor t\rfloor=\max \{n \in \mathbb{N}: n \leq t\}$ is the integer part of $t$, so $\lim _{t \rightarrow \infty} \int_{0}^{t} y(s) d s=0$. Therefore, $y$ is Riemann integrable. Invoking Proposition 4.2(c), we conclude that $y$ is weakly meagre. Alternatively, the weak meagreness of $y$ can be established by a straightforward verification of the defining property of weak meagreness. However, $y$ is not meagre, as the following argument shows. From the observation that $\left|y_{n}(t)\right|=1$ for all $t$ in the set $[n+1 /(5 n), n+2 /(5 n)] \cup[n+3 /(5 n), n+4 /(5 n)]$ it follows that

$$
\mu\left(\left\{t \in \mathbb{R}_{+}:|y(t)| \geq 1\right\}\right)=\sum_{n \in \mathbb{N}} \frac{2}{5 n}=\infty,
$$

confirming that $y$ is not meagre.

Example 7.2. We construct a continuous meagre function $y$ such that for each $\tau>0$ the integral $\int_{t}^{t+\tau} y(s) d s$ does not converge to 0 as $t \rightarrow \infty$.

Consider the continuous nonnegative function $y: \mathbb{R}_{+} \rightarrow \mathbb{R}$ given by

$$
t \mapsto \sum_{n \in \mathbb{N}} y_{n}(t)
$$

where $y_{n}: \mathbb{R}_{+} \rightarrow\left[0, n^{2}\right]$ is the piecewise linear continuous function that is compactly supported on $\left[n, n+1 / n^{2}\right]$ and has the graph as shown in Figure 7 (wherein the corners occur at $t=n+k /\left(2 n^{2}\right)$ for $\left.k=0,1,2\right)$.

For any $\lambda>0$ we have

$$
\mu\left(\left\{t \in \mathbb{R}_{+}:|y(t)| \geq \lambda\right\}\right) \leq \sum_{n=1}^{\infty} 1 / n^{2}<\infty,
$$




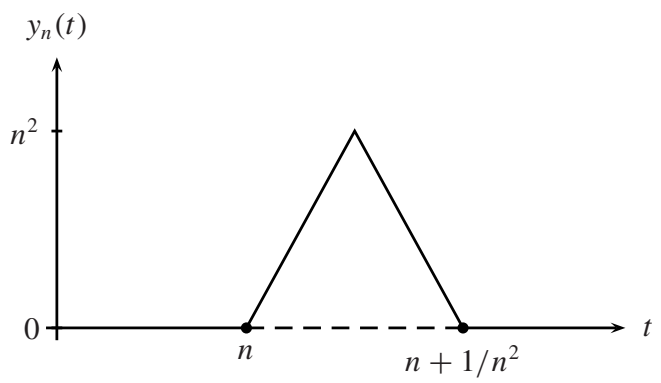

Figure 7.

showing that $y$ is meagre (so a fortiori weakly meagre). However, for any $\tau>0$ we have

$$
\int_{n}^{n+\tau} y(t) d t \geq \int_{n}^{n+1 / n^{2}} y_{n}(t) d t=\frac{1}{2}
$$

for all $n$ in $\mathbb{N}$ with $n \geq 1 / \sqrt{\tau}$, so $\int_{t}^{t+\tau} y(s) d s$ does not converge to 0 as $t \rightarrow \infty$.

\section{REFERENCES}

1. H. Amann, Ordinary Differential Equations: An Introduction to Nonlinear Analysis, Walter de Gruyter, Berlin, 1990.

2. J. P. Aubin and A. Cellina, Differential Inclusions, Springer-Verlag, Berlin, 1984.

3. I. Barbălat, Systèmes d'équations différentielles d'oscillations non linéaires, Revue de Mathématiques Pures et Appliquées IV (1959) 267-270.

4. G. D. Birkhoff, Dynamical Systems, Colloquium Publications, no. 9, American Mathematical Society, Providence, 1927.

5. C. I. Byrnes and C. F. Martin, An integral-invariance principle for nonlinear systems, IEEE Trans. Automatic Control AC-40 (1995) 983-994.

6. F. H. Clarke, Optimization and Nonsmooth Analysis, Wiley, New York, 1983.

7. F. H. Clarke, Yu. S. Ledyaev, R. J. Stern, and P. R. Wolenski, Nonsmooth Analysis and Control Theory, Springer-Verlag, New York, 1998.

8. E. A. Coddington and N. Levinson, Theory of Ordinary Differential Equations, McGraw-Hill, New York, 1955.

9. C. Corduneanu, Integral Equations and Stability of Feedback Systems, Academic Press, New York, 1973.

10. K. Deimling, Multivalued Differential Equations, Walter de Gruyter, Berlin, 1992.

11. W. Desch, H. Logemann, E. P. Ryan, and E. D. Sontag, Meagre functions and asymptotic behaviour of dynamical systems, Nonlinear Analysis: Theory, Methods \& Applications 44 (2001) 1087-1109.

12. A. F. Filippov, Differential Equations with Discontinuous Righthand Sides, Kluwer, Dordrecht, 1988.

13. W. Hahn, Stability of Motion, Springer-Verlag, Berlin, 1967.

14. P. R. Halmos, Measure Theory, Springer-Verlag, New York, 1974.

15. R. E. Kalman and J. E. Bertram, Control systems analysis and design via the "second method" of Lyapunov; part 1: continuous-time systems, Trans. ASME 82 (1960) 371-393.

16. H. W. Knobloch and F. Kappel, Gewöhnliche Differentialgleichungen, B. G. Teubner, Stuttgart, 1974.

17. J. P. LaSalle, The extent of asymptotic stability, Proc. Nat. Acad. Sci. USA 46 (1960) 363-365.

18. - Some extensions of Liapunov's Second Method, IRE Trans. Circuit Theory CT-7 (1960) 520527.

19. - Stability theory for ordinary differential equations, J. Differential Equations 4 (1968) 57-65.

20. - The Stability of Dynamical Systems, SIAM, Philadelphia, 1976.

21. E. H. Lieb and M. Loss, Analysis, American Mathematical Society, Providence, 1997.

22. H. Logemann and E. P. Ryan, Non-autonomous systems: Asymptotic behaviour and weak invariance principles, J. Differential Equations 189 (2003) 440-460.

23. A. M. Lyapunov, Problème général de la stabilité du mouvement, Ann. Fac. Sci. Toulouse 9 (1907) 203 474; reprinted in Ann. Math. Study, no. 17, Princeton University Press, Princeton, 1949. 
24. A. M. Lyapunov, The general problem of the stability of motion (trans. A. T. Fuller), Int. J. Control 55 (1992) 531-773.

25. V. M. Popov, Hyperstability of Control Systems, Springer-Verlag, Berlin, 1973.

26. E. P. Ryan, Discontinuous feedback and universal adaptive stabilization, in Control of Uncertain Systems, D. Hinrichsen and B. Mårtensson, eds., Birkhäuser, Boston, 1990, pp. 245-258.

27. - An integral invariance principle for differential inclusions with application in adaptive control, SIAM J. Control \& Optim. 36 (1998) 960-980.

28. S. Sastry, Nonlinear Systems: Analysis, Stability and Control, Springer-Verlag, New York, 1999.

29. G. V. Smirnov, Introduction to the Theory of Differential Inclusions, American Mathematical Society, Providence, 2002.

30. A. R. Teel, Asymptotic convergence from $L_{p}$ stability, IEEE Trans. Auto. Control AC-44 (1999) 21692170 .

31. W. Walter, Ordinary Differential Equations, Springer-Verlag, New York, 1998.

32. J. Zabczyk, Mathematical Control Theory: An Introduction, Birkhäuser, Boston, 1992.

HARTMUT LOGEMANN received his Ph.D. from the University of Bremen (Germany) under the guidance of Diederich Hinrichsen. He teaches and conducts research at the University of Bath (in the Southwest of England). His research interests are in mathematical systems and control theory with particular emphasis on the control of infinite-dimensional systems. His outside interests include jazz and literature.

Department of Mathematical Sciences, University of Bath, Bath, BA2 7AY, UK

hl@maths.bath.ac.uk

EUGENE P. RYAN received his Ph.D. from the University of Cambridge (England). He teaches and conducts research at the University of Bath. His research interests are in mathematical systems and control theory with particular emphasis on nonlinearity.

Department of Mathematical Sciences, University of Bath, Bath, BA2 7AY, UK

epr@maths.bath.ac.uk 